\title{
American Society of Hematology 2018 guidelines for management of venous thromboembolism: diagnosis of venous thromboembolism
}

\author{
Wendy Lim, ${ }^{1,2}$ Grégoire Le Gal, ${ }^{3}$ Shannon M. Bates, ${ }^{1,4}$ Marc Righini, ${ }^{5,6}$ Linda B. Haramati, ${ }^{7,8}$ Eddy Lang, ${ }^{9,10}$ Jeffrey A. Kline, ${ }^{11}$ \\ Sonja Chasteen, ${ }^{12}$ Marcia Snyder, ${ }^{13}$ Payal Patel, ${ }^{14}$ Meha Bhatt, ${ }^{15}$ Parth Patel, ${ }^{16}$ Cody Braun, ${ }^{17}$ Housne Begum, ${ }^{15}$ Wojtek Wiercioch, ${ }^{15}$ \\ Holger J. Schünemann, ${ }^{1,15}$ and Reem A. Mustafa ${ }^{15,18}$ \\ ${ }^{1}$ Department of Medicine and ${ }^{2}$ Department of Pathology \& Molecular Medicine, McMaster University, Hamilton, ON, Canada; ${ }^{3}$ Department of Medicine, University of Ottawa/ \\ Ottawa Hospital Research Institute, Ottawa, ON, Canada; ${ }^{4}$ Thrombosis and Atherosclerosis Research Institute (TaARI), McMaster University, Hamilton, ON, Canada; ${ }^{5}$ Division \\ of Angiology and Hemostasis, Department of Medical Specialties, Geneva University Hospitals, Geneva, Switzerland; ${ }^{6}$ Faculty of Medicine, University of Geneva, Geneva, \\ Switzerland; ${ }^{7}$ Department of Radiology and ${ }^{8}$ Department of Medicine, Montefiore Medical Center/College of Medicine, Albert Einstein, Bronx, NY; ${ }^{9}$ Department of Emergency \\ Medicine, Cumming School of Medicine, University of Calgary, Calgary, AB, Canada; ${ }^{10}$ Rockyview General Hospital, Calgary, AB, Canada; ${ }^{11}$ Department of Emergency \\ Medicine, School of Medicine, Indiana University, Indianapolis, IN; ${ }^{12}$ Fort Myers, FL; ${ }^{13}$ Wheaton, IL; ${ }^{14}$ Department of Emergency Medicine, University of Illinois at Chicago, \\ Chicago, IL; ${ }^{15}$ Department of Health Research Methods, Evidence, and Impact, McMaster University, Hamilton, ON, Canada; ${ }^{16}$ Department of Internal Medicine, School of \\ Medicine, University of Missouri-Kansas City, Kansas City, MO; ${ }^{17}$ Department of Medicine, Loyola University Medical Center, Maywood, IL; and ${ }^{18}$ Division of Nephrology and \\ Hypertension, Department of Medicine, University of Kansas Medical Center, Kansas City, KS
}

Background: Modern diagnostic strategies for venous thromboembolism (VTE) incorporate pretest probability (PTP; prevalence) assessment. The ability of diagnostic tests to correctly identify or exclude VTE is influenced by VTE prevalence and test accuracy characteristics.

Objective: These evidence-based guidelines are intended to support patients, clinicians, and health care professionals in VTE diagnosis. Diagnostic strategies were evaluated for pulmonary embolism (PE), deep vein thrombosis (DVT) of the lower and upper extremity, and recurrent VTE.

Methods: The American Society of Hematology (ASH) formed a multidisciplinary panel including patient representatives. The McMaster University GRADE Centre completed systematic reviews up to 1 October 2017. The panel prioritized questions and outcomes and used the Grading of Recommendations Assessment, Development and Evaluation (GRADE) approach to assess evidence and make recommendations. Test accuracy estimates and VTE population prevalence were used to model expected outcomes in diagnostic pathways. Where modeling was not feasible, management and accuracy studies were used to formulate recommendations.

Results: Ten recommendations are presented, by PTP for patients with suspected PE and lower extremity DVT, and for recurrent VTE and upper extremity DVT.

Conclusions: For patients at low (unlikely) VTE risk, using D-dimer as the initial test reduces the need for diagnostic imaging. For patients at high (likely) VTE risk, imaging is warranted. For PE diagnosis, ventilationperfusion scanning and computed tomography pulmonary angiography are the most validated tests, whereas lower or upper extremity DVT diagnosis uses ultrasonography. Research is needed on new diagnostic modalities and to validate clinical decision rules for patients with suspected recurrent VTE.

\section{Summary of recommendations}

These guidelines are based on updated and original systematic reviews of evidence conducted by researchers and developed under the direction of the McMaster University GRADE Centre and the University of Kansas Medical Center, with international collaborators. The panel followed best practices for guideline development recommended by the Institute of Medicine (now National Academy of Medicine) and the Guidelines International Network (GIN). ${ }^{1-4}$ The panel used the GRADE approach ${ }^{5,6}$ to assess quality of evidence and formulate recommendations.

Accurate diagnosis of venous thromboembolism (VTE) is important due to the morbidity and mortality associated with missed diagnoses and the potential side effects, patient inconvenience and resource

Submitted 19 August 2018; accepted 2 October 2018. DOI 10.1182/ bloodadvances. 2018024828

Resources for implementing these guidelines, including apps, patient decision aids, and teaching slide sets, may be accessed at the ASH web page hematology.org/vte.
The full-text version of this article contains a data supplement.

(c) 2018 by The American Society of Hematology 
implications of anticoagulant treatment given for VTE. Diagnostic strategies for VTE are based on assessment of the pretest probability (PTP) for individual patients, which provides an estimate of the expected prevalence of VTE at a population level. Validated clinical decision rules to determine PTP have been published and their use is recommended in the initial assessment of patients with suspected VTE. ${ }^{7}$ The scope of this guideline does not include a review of existing clinical decision rules or comparisons between different rules. It is assumed the user will use a validated method to determine PTP. For patients with suspected pulmonary embolism $(\mathrm{PE})$, this guideline assumed the prevalence of $\mathrm{PE}$ for patients with low, intermediate, and high PTP to be $\leq 5 \%, 20 \%$ ( $\pm 10 \%)$, and $\geq 50 \%$, respectively. For patients with suspected deep vein thrombosis (DVT) of the lower extremities, the prevalence of DVT for patients with low, intermediate and high PTP was estimated at $\leq 10 \%, 25 \%$ ( $\pm 10 \%$ ), and $\geq 50 \%$, respectively. For patients with suspected upper extremity DVT, the prevalence for patients with likely and unlikely probability was estimated at $10 \%$ and $40 \%$.

Diagnostic tests for VTE are subject to error and the ability of these tests to accurately diagnose VTE is dependent on the prevalence of VTE in the population. The rate of true-positive (TP; patients correctly identified as having VTE), true-negative (TN; patients correctly identified as not having VTE), false-positive (FP; patients incorrectly identified as having VTE), and false-negative (FN; patients incorrectly identified as not having VTE) test results can be determined for a single diagnostic test or 1 or more tests in a diagnostic strategy, where the results of the prior test influence the performance of a subsequent test. Determining the optimal diagnostic strategy is based on defining acceptable "misdiagnosis" rates of FN and FP results based on patients' and physicians' values and preferences, risk associated with different tests, cost, and feasibility. These guidelines provide recommendations on the optimal diagnostic strategy for suspected VTE based on prevalence of disease, and present acceptable alternate diagnostic strategies accounting for the variability in different practice settings.

\section{Interpretation of strong and conditional recommendations}

The strength of a recommendation is expressed as either strong ("the guideline panel recommends..."), or conditional ("the guideline panel suggests...") and has the following interpretation:

\section{Strong recommendation}

- For patients: Most individuals in this situation would want the recommended course of action, and only a small proportion would not.

- For clinicians: Most individuals should receive the intervention or test. Formal decision aids are not likely to be needed to help individual patients make decisions consistent with their values and preferences.

- For policy makers: The recommendation can be adopted as policy in most situations. Adherence to this recommendation according to the guideline could be used as a quality criterion or performance indicator.

- For researchers: The recommendation is supported by credible research or other convincing judgments that make additional research unlikely to alter the recommendation. On occasion, a strong recommendation is based on low or very low certainty in the evidence. In such instances, further research may provide important information that alters the recommendation.

\section{Conditional recommendation}

- For patients: The majority of individuals in this situation would want the suggested course of action, but many would not. Decision aids may be useful in helping patients to make decisions consistent with their individual risks, values, and preferences.

- For clinicians: Different choices will be appropriate for individual patients, and clinicians must help each patient arrive at a management decision consistent with the patient's values and preferences. Decision aids may be useful in helping individuals to make decisions consistent with their individual risks, values, and preferences.

- For policy makers: Policy-making will require substantial debate and involvement of various stakeholders. Performance measures about the suggested course of action should focus on whether an appropriate decision-making process is duly documented.

- For researchers: This recommendation is likely to be strengthened (for future updates or adaptation) by additional research. An evaluation of the conditions and criteria (and the related judgments, research evidence, and additional considerations) that determined the conditional (rather than strong) recommendation will help identify possible research gaps.

\section{Recommendations}

\section{Diagnosis of PE}

Low PTP/prevalence ( $\mathbf{5}$ 5\%). Recommendation 1a. The American Society of Hematology (ASH) guideline panel recommends using a strategy starting with D-dimer for excluding $\mathrm{PE}$ in a population with low prevalence/PTP ( $\leq 5 \%$ ), followed by ventilation-perfusion (VQ) scan or computed tomography pulmonary angiography (CTPA) for patients requiring additional testing. If $\mathrm{D}$-dimer is not readily available, alternate acceptable strategies include performing VQ scan or CTPA alone. (Strong recommendation for D-dimer based on high certainty in the evidence of effects on clinical outcomes $\oplus \oplus \oplus \oplus$ and moderate certainty in the evidence of diagnostic accuracy studies $\oplus \oplus \oplus \bigcirc$; conditional recommendation for VQ scan or CTPA based on very low certainty in the evidence of effects on clinical outcomes $\oplus \bigcirc \bigcirc \bigcirc$ and low certainty in the evidence from diagnostic accuracy studies $\oplus \oplus \bigcirc)$ ).

\section{Remarks:}

- For patients presenting to an emergency department with a low probability of $\mathrm{PE}$, the Pulmonary Embolism Rule-out Criteria (PERC) may be used to determine whether D-dimer testing is warranted.

- Validated clinical decision rules were used to assess clinical probability of PE in studies evaluating different diagnostic strategies for patients suspected of having a first episode PE. The Geneva score has been validated only in an outpatient population. If a 2-level clinical decision rule is used, this recommendation corresponds to the "unlikely PE" category. A decision to start with $D$-dimer assumes the results will be obtained in a timely manner and that the cost of D-dimer screening is offset by avoiding unnecessary VQ or CTPA for patients at low PTP for PE. If the D-dimer strategy is followed, a highly sensitive $\mathrm{D}$-dimer assay is required. A negative D-dimer rules out $\mathrm{PE}$ and no additional testing or anticoagulation is 
required. D-dimer has limited utility in hospitalized patients and in certain patient populations (postsurgical, pregnant) due to the high frequency of positive D-dimer results with standard thresholds.

- Use of an age-adjusted D-dimer cutoff in outpatients older than 50 years is as safe as the standard cutoff and increases the diagnostic utility of the test. Age-adjusted cutoff = age (years) $\times$ $10 \mu \mathrm{g} / \mathrm{L}$ (using D-dimer assays with a cutoff of $500 \mu \mathrm{g} / \mathrm{L}$ ).

- VQ scan is preferred over CTPA as the subsequent test to limit radiation exposure for patients likely to have a diagnostic scan, and in centers where VQ scans are available with expertise to interpret the results in a timely manner. CTPA is preferred when VQ scan is not feasible.

- The strategy assumes that test results are obtained under optimal conditions. Suboptimal D-dimer or CTPA results may require repeat testing or an alternate strategy. If the VQ scan is nondiagnostic, additional testing with proximal ultrasound of the lower extremities or CTPA should be considered.

Recommendation $1 \mathrm{~b}$. The ASH guideline panel recommends against using a positive D-dimer alone to diagnose $\mathrm{PE}$, and against additional testing following negative CTPA or normal VQ scan in a population with low prevalence/PTP $(\leq 5 \%)$. (See grading for recommendation 1a.)

Intermediate PTP/prevalence ( 20\%). Recommendation 2a. The ASH guideline panel suggests using a strategy starting with D-dimer for excluding $\mathrm{PE}$ in a population with intermediate prevalence/PTP ( 20\%), followed by VQ scan or CTPA for patients requiring additional testing. If $\mathrm{D}$-dimer is not readily available, alternate acceptable strategies include performing VQ scan or CTPA alone. Patients who are likely to have a nondiagnostic VQ scan should undergo CTPA. (Conditional recommendation for D-dimer based on high certainty in the evidence of effects on clinical outcomes $\oplus \oplus \oplus \oplus$ and moderate certainty in the evidence about diagnostic accuracy studies $\oplus \oplus \oplus \mathrm{O}$; conditional recommendation for VQ scan or CTPA based on very low certainty in the evidence of effects on clinical outcomes $\oplus 00 \bigcirc$ and moderate certainty in the evidence from diagnostic accuracy studies $\oplus \oplus \oplus \bigcirc)$.

\section{Remarks:}

- Validated clinical decision rules were used to assess clinical probability of $\mathrm{PE}$ in studies evaluating different diagnostic strategies for patients suspected of having a first episode PE. The Geneva score has been validated only in an outpatient population.

- A decision to start with D-dimer assumes the results will be obtained in a timely manner and that the cost of D-dimer screening is offset by avoiding unnecessary VQ or CTPA for patients at intermediate PTP for PE. If the D-dimer strategy is followed, a highly sensitive D-dimer assay is required. A negative $D$-dimer rules out $P E$ and no additional testing or anticoagulation is required. D-dimer has limited utility in hospitalized patients and in certain patient populations (postsurgical, pregnant) due to the high frequency of positive D-dimer results with standard thresholds.

- Use of an age-adjusted D-dimer cutoff in outpatients older than 50 years is as safe as the standard cutoff and increases the diagnostic utility of the test. Age-adjusted cutoff = age (years) $\times$ $10 \mu \mathrm{g} / \mathrm{L}$ (using D-dimer assays with a cutoff of $500 \mu \mathrm{g} / \mathrm{L}$ ).
- VQ scan is preferred over CTPA as the subsequent test to limit radiation exposure for patients likely to have a diagnostic scan, and in centers where VQ scans are available with expertise to interpret the results in a timely manner. CTPA is preferred when VQ scan is not feasible.

- The strategy assumes that test results are obtained under optimal conditions. Suboptimal D-dimer or CTPA results may require repeat testing or an alternate strategy. If the $\mathrm{VQ}$ scan is nondiagnostic, additional testing with CTPA should be considered.

Recommendation $2 b$. The $\mathrm{ASH}$ guideline panel recommends against using a positive D-dimer alone to diagnose $\mathrm{PE}$, and against additional testing following negative CTPA or normal VQ scan in a population with intermediate prevalence/PTP $(\sim 20 \%)$. (See grading for recommendation 2a.)

High PTP/prevalence ( $\mathbf{5 0 \%}$ ). Recommendation 3a. The ASH guideline panel suggests using a strategy starting with CTPA for assessing patients suspected of having $P E$ in a population with high prevalence/PTP $(\geq 50 \%$ ). (Conditional recommendation for CTPA based on very low certainty in the evidence of effects on clinical outcomes $\oplus \bigcirc \bigcirc \bigcirc$ and moderate certainty in the evidence of diagnostic accuracy studies $\oplus \oplus \oplus \bigcirc)$.

\section{Remarks:}

- Validated clinical decision rules were used to assess clinical probability of PE in studies evaluating different diagnostic strategies for patients suspected of having a first episode PE. The Geneva score has been validated only in an outpatient population. If a 2-level clinical decision rule is used, this recommendation corresponds to the "likely PE" category.

- The strategy assumes that test results are obtained under optimal conditions. Suboptimal CTPA results may require repeat testing.

- If CTPA is not feasible (eg, contrast dye allergy, renal impairment, unavailability), VQ scan may be acceptable if nondiagnostic scans are followed by additional testing.

- In cases where clinical suspicion for PE remains high with a negative initial CTPA, additional testing with VQ scan or proximal ultrasound of the lower extremities may be considered.

Recommendation $3 b$. The ASH guideline panel recommends against using a positive D-dimer alone to diagnose PE, and against using D-dimer as a subsequent test following a negative CT scan in a population with high prevalence/PTP $(\geq 50 \%)$. (See grading for recommendation 3a.)

Recurrent PE. Recommendation 4. The ASH guideline panel suggests using a strategy starting with D-dimer for excluding recurrent PE in a population with unlikely PTP. Patients with a positive D-dimer or those who have a likely PTP should undergo CTPA. (Conditional recommendation for D-dimer and CTPA based on low certainty in the evidence of effects on clinical outcomes $\oplus \oplus \bigcirc \bigcirc$ and moderate certainty in the evidence from diagnostic accuracy studies $\oplus \oplus \oplus \bigcirc)$.

\section{Remarks:}

- In studies assessing this diagnostic strategy for patients suspected of having recurrent PE, Wells and Geneva scores were used to assess clinical probability of recurrent PE. Previous VTE is a predictor in these scores, but the Wells and Geneva scores have 
not been specifically validated for patients with suspected recurrent $\mathrm{PE}$.

- A decision to start with D-dimer assumes the results will be obtained in a timely manner and that the cost of D-dimer screening is offset by avoiding unnecessary VQ or CTPA for patients at unlikely PTP for recurrent PE. If the D-dimer strategy is followed, a highly sensitive $D$-dimer assay is required. A negative D-dimer rules out $\mathrm{PE}$ and no additional testing or anticoagulation is required. D-dimer has limited utility in hospitalized patients and in certain patient populations (postsurgical, pregnant) due to the high frequency of positive D-dimer results with standard thresholds. There is a limited data on the utility of D-dimer for patients receiving anticoagulant therapy who present with suspected recurrent PE.

- The strategy assumes that test results are obtained under optimal conditions. Suboptimal CTPA results may require repeat testing.

- If prior imaging is available, comparison of the previous and current imaging is warranted to determine whether the findings are new and represent recurrent PE.

\section{Diagnosis of lower extremity DVT}

Low PTP/prevalence (s10\%). Recommendation 5a. The ASH guideline panel recommends using a strategy starting with D-dimer for excluding DVT in a population with low prevalence/ PTP ( $\leq 10 \%)$, followed by proximal lower extremity ultrasound or whole-leg ultrasound for patients requiring additional testing. If D-dimer is not readily available, alternate acceptable strategies include performing proximal lower extremity or whole-leg ultrasound alone. (Strong recommendation for D-dimer based on moderate certainty in the evidence of effects on clinical outcomes $\oplus \oplus \oplus \bigcirc$ and moderate certainty in the evidence of diagnostic accuracy studies $\oplus \oplus \oplus \bigcirc$; conditional recommendation for proximal or whole-leg ultrasound based on very low certainty in the evidence of effects on clinical outcomes $\oplus \circ \bigcirc \bigcirc$ and moderate certainty in the evidence from diagnostic accuracy studies $\oplus \oplus \oplus \bigcirc)$.

\section{Remarks:}

- Validated clinical decision rules were used to assess clinical probability of DVT in studies evaluating different diagnostic strategies for patients suspected of having a first episode DVT. If a 2-level clinical decision rule is used, this recommendation corresponds to the "unlikely DVT" category.

- A decision to start with D-dimer assumes the results will be obtained in a timely manner and that the cost of D-dimer screening is offset by avoiding unnecessary proximal or wholeleg ultrasound for patients at low PTP for DVT. If the D-dimer strategy is followed, a highly sensitive $D$-dimer assay is required. A negative D-dimer rules out DVT and no additional testing or anticoagulation is required. D-dimer has limited utility in hospitalized patients and in certain patient populations (postsurgical, pregnant) due to the high frequency of positive D-dimer results with standard thresholds.

- The strategy assumes that test results are obtained under optimal conditions. Suboptimal D-dimer or ultrasound results may require repeat testing.
Recommendation $5 \mathrm{~b}$. The $\mathrm{ASH}$ guideline panel recommends against using a positive D-dimer alone to diagnose DVT, and against additional testing following negative proximal or whole-leg ultrasound in a population with low prevalence/PTP $(\leq 10 \%)$. (See grading for recommendation 5a.)

Intermediate PTP/prevalence ( 25\%). Recommendation 6a. The ASH guideline panel suggests using a strategy using whole-leg ultrasound, or starting with proximal lower extremity ultrasound for evaluating patients suspected of having DVT in a population with intermediate prevalence/PTP ( $\sim 25 \%)$. No further testing is required if the whole-leg ultrasound is negative, but a negative initial proximal ultrasound should be followed by serial proximal ultrasound if no alternative diagnosis is identified. In an intermediate PTP population where the prevalence is lower, other potentially acceptable strategies include proximal lower extremity ultrasound alone with no additional follow-up testing for negative results, or a strategy starting with D-dimer for excluding DVT followed by proximal lower extremity ultrasound or whole-leg ultrasound for patients requiring additional testing. (Conditional recommendation for proximal or whole-leg ultrasound based on very low certainty in the evidence of effects on clinical outcomes $\oplus \bigcirc \bigcirc \bigcirc$ and high certainty in the evidence from diagnostic accuracy studies $\oplus \oplus \oplus \oplus$; conditional recommendation for D-dimer based on moderate certainty in the evidence of effects on clinical outcomes $\oplus \oplus \oplus \bigcirc$ and moderate certainty in the evidence from diagnostic accuracy studies $\oplus \oplus \oplus \bigcirc)$.

\section{Remarks:}

- Validated clinical decision rules were used to assess clinical probability of DVT in studies evaluating different diagnostic strategies for patients suspected of having a first episode DVT.

- Studies where patients are determined to have an intermediate PTP for DVT have prevalence estimates of $\sim 13 \%$ to $24 \% .^{8} \mathrm{~A}$ decision to use a D-dimer strategy in an intermediate PTP population assumes the prevalence is $\sim 15 \%$ or less. A decision to start with D-dimer assumes the results will be obtained in a timely manner and that the cost of D-dimer screening is offset by avoiding unnecessary ultrasound for patients at intermediate PTP for DVT. If the D-dimer strategy is followed, a highly sensitive D-dimer assay is required. A negative D-dimer rules out DVT and no additional testing or anticoagulation is required. D-dimer has limited utility in hospitalized patients and in certain patient populations (postsurgical, pregnant) due to the high frequency of positive D-dimer results with standard thresholds.

- The strategy assumes that test results are obtained under optimal conditions. Suboptimal D-dimer or ultrasound results may require repeat testing.

- Serial ultrasound is defined as 1 additional ultrasound in 1 week following the initial ultrasound.

Recommendation $6 b$. The ASH guideline panel recommends against using a positive D-dimer alone to diagnose DVT in a population with intermediate prevalence/PTP ( 25\%). (See grading for recommendation 6a.)

High PTP/prevalence ( $\mathbf{5 0 \%}$ ). Recommendation 7a. The ASH guideline panel suggests using a strategy starting with proximal lower extremity or whole-leg ultrasound for assessing patients suspected of having DVT in a population with high prevalence/PTP $(\geq 50 \%)$. This 
should be followed by serial ultrasound if the initial ultrasound is negative and no alternative diagnosis is identified. (Conditional recommendation for proximal or whole-leg ultrasound based on very low certainty in the evidence of effects on clinical outcomes $\oplus \bigcirc \bigcirc \bigcirc$ and high certainty in the evidence from diagnostic accuracy studies $\oplus \oplus \oplus \oplus)$.

\section{Remarks:}

- Validated clinical decision rules were used to assess clinical probability of DVT in studies evaluating different diagnostic strategies for patients suspected of having a first episode DVT. If a 2-level clinical decision rule is used, this recommendation corresponds to the "likely DVT" category.

- The strategy assumes that test results are obtained under optimal conditions. Suboptimal ultrasound results may require repeat testing.

Recommendation $7 \mathrm{~b}$. The $\mathrm{ASH}$ guideline panel recommends against using a positive D-dimer alone to diagnose DVT in a population with high prevalence/PTP $(\geq 50 \%$ ). (See grading for recommendation $7 \mathrm{a}$.)

Recurrent DVT (lower extremity). Recommendation 8. The ASH guideline panel suggests using a strategy starting with D-dimer for excluding recurrent DVT in a population with unlikely PTP. Patients with positive D-dimer or those who have likely PTP should undergo proximal lower extremity ultrasound. (Conditional recommendation for D-dimer and ultrasound based on low certainty in the evidence of effects on clinical outcomes $\oplus \oplus \bigcirc \bigcirc$ and low certainty in the evidence from diagnostic accuracy studies $\oplus \oplus \bigcirc \bigcirc)$.

\section{Remarks:}

- In studies assessing this diagnostic strategy for patients suspected of having recurrent DVT, a modified Wells score was used to assess clinical probability of suspected recurrent DVT.

- A decision to start with D-dimer assumes the results will be obtained in a timely manner and that the cost of D-dimer screening is offset by avoiding unnecessary ultrasound for patients at unlikely PTP for DVT. If the D-dimer strategy is followed, a highly sensitive $D$-dimer assay is required. A negative D-dimer rules out DVT and no additional testing or anticoagulation is required. D-dimer has limited utility in hospitalized patients and in certain patient populations (postsurgical, pregnant) due to the high frequency of positive D-dimer results with standard thresholds. There are limited data on the utility of D-dimer for patients receiving anticoagulant therapy who present with suspected recurrent DVT.

- The strategy assumes that test results are obtained under optimal conditions. Suboptimal D-dimer or ultrasound results may require repeat testing.

- If clinical suspicion remains high following a negative initial proximal ultrasound, serial proximal ultrasound could be considered.

- If prior imaging is available, comparison of the previous and current imaging is warranted to determine whether the findings are new and represent recurrent DVT. Studies evaluating compression ultrasound findings of recurrent DVT generally consider involvement of a new venous segment or increase noncompressibility of $>4 \mathrm{~mm}$ as diagnostic of recurrent DVT.

\section{Diagnosis of upper extremity DVT}

Unlikely PTP/prevalence (10\%). Recommendation 9a. The ASH guideline panel suggests a strategy starting with D-dimer for excluding upper extremity DVT in a population with low prevalence/ unlikely PTP (10\%), followed by duplex ultrasound if D-dimer is positive. If $\mathrm{D}$-dimer is not readily available, performing duplex ultrasound alone is acceptable. (Conditional recommendation for D-dimer based on very low certainty in the evidence of effects on clinical outcomes $\oplus \bigcirc \bigcirc \bigcirc$ and moderate certainty in the evidence from diagnostic accuracy studies $\oplus \oplus \oplus \bigcirc$; conditional recommendation for duplex ultrasound based on very low certainty in the evidence of effects on clinical outcomes $\oplus \bigcirc \bigcirc \bigcirc$ and low certainty in the evidence from diagnostic accuracy studies $\oplus \oplus \bigcirc)$ ).

\section{Remarks:}

- In studies assessing this diagnostic strategy for patients suspected of having upper extremity DVT, a dichotomized Constans score (where score $\leq 1$ is unlikely and $\geq 2$ is likely) was used to assess clinical probability of suspected upper extremity DVT.

- A decision to start with D-dimer assumes the results will be obtained in a timely manner and that the cost of D-dimer screening is offset by avoiding unnecessary duplex ultrasound for patients at unlikely PTP for upper extremity DVT. If the $\mathrm{D}$-dimer strategy is followed, a highly sensitive D-dimer assay is required. A negative D-dimer rules out upper extremity DVT and no additional testing or anticoagulation is required. D-dimer has limited utility in hospitalized patients and in certain patient populations (postsurgical, pregnancy) due to the high frequency of positive D-dimer results with standard thresholds.

- The strategy assumes that test results are obtained under optimal conditions. Suboptimal D-dimer or duplex ultrasound results may require repeat testing.

- If clinical suspicion remains high following a negative initial duplex ultrasound, serial duplex ultrasound could be considered.

Recommendation 9b. The ASH guideline panel recommends against using a positive D-dimer alone to diagnose upper extremity DVT in a population with low prevalence/unlikely PTP (10\%). (See grading for recommendation 9a.)

Likely PTP/prevalence (40\%). Recommendation 10a. The ASH guideline panel suggests a strategy of either D-dimer followed by duplex ultrasound/serial duplex ultrasound, or duplex ultrasound/ serial duplex ultrasound alone for assessing patients suspected of having upper extremity DVT in a population with high prevalence/ likely PTP (40\%). (Conditional recommendation for D-dimer based on very low certainty in the evidence of effects on clinical outcomes $\oplus \bigcirc \bigcirc \bigcirc$ and moderate certainty in the evidence from diagnostic accuracy studies $\oplus \oplus \oplus \bigcirc$; conditional recommendation for duplex ultrasound based on very low certainty in the evidence of effects on clinical outcomes $\oplus \bigcirc \bigcirc \bigcirc$ and low certainty in the evidence from diagnostic accuracy studies $\oplus \oplus \bigcirc \bigcirc)$.

\section{Remarks:}

- In studies assessing this diagnostic strategy for patients suspected of having upper extremity DVT, a dichotomized Constans score (where score $\leq 1$ is unlikely and $\geq 2$ is likely) was used to assess clinical probability of suspected upper extremity DVT. 
- A decision to start with D-dimer assumes the results will be obtained in a timely manner and that the cost of D-dimer screening is offset by avoiding unnecessary duplex ultrasound. If the D-dimer strategy is followed, a highly sensitive D-dimer assay is required. A negative D-dimer rules out upper extremity DVT and no additional testing or anticoagulation is required. D-dimer has limited utility in hospitalized patients and in certain patient populations (postsurgical, pregnant) due to the high frequency of positive D-dimer results with standard thresholds.

- The strategy assumes that test results are obtained under optimal conditions. Suboptimal D-dimer or duplex ultrasound results may require repeat testing.

Recommendation 10b. The ASH guideline panel recommends against using a positive D-dimer alone to diagnose upper extremity DVT in a population with high prevalence/likely PTP (40\%). (See grading for recommendation 10a.)

\section{Values and preferences}

The ASH panel including the patient representatives placed higher value on minimizing radiation exposure to the patient if the diagnosis could be obtained using alternate methods with less radiation exposure. The panel also placed higher value on reducing the number of tests in a diagnostic strategy to obtain a diagnosis if the same results could be achieved. Although a single imaging test can be used to establish the diagnosis of VTE in low PTP populations, a strategy using D-dimer as the initial test is recommended to decrease radiation exposure and/or exposure to diagnostic imaging.

\section{Explanations and other considerations}

These recommendations assume the D-dimer results will be obtained in a timely manner and that the inconvenience and cost of D-dimer screening is acceptable to patients to potentially avoid undergoing additional diagnostic testing. It also assumes the costs of D-dimer screening will be offset by avoiding unnecessary diagnostic imaging.

Suboptimal conditions may also require repeat tests. Examples include insufficient blood draw when measuring D-dimer, motion artifact or improper timing of contrast dye resulting in insufficient opacification of the pulmonary arteries on CTPA, or VQ performed for patients with preexisting lung disease.

Additionally, these recommendations acknowledge that diagnostic test availability and expertise in interpretation varies in different settings. For example, although VQ scan may be preferred over CTPA as the subsequent test after a positive D-dimer to limit radiation exposure, this assumes the availability of technicians and expertise to obtain and interpret the results in a timely manner (feasibility). This also assumes the VQ scan will result in a diagnostic result (ie, normal or high probability). Patients who are older or have preexisting lung disease are more likely to have a nondiagnostic VQ scan. Last, modern CT scanners are increasingly able to detect small thrombi (eg, subsegmental PE) where the clinical relevance of such findings remains unclear and may result in unnecessary anticoagulant treatment. Assessing the PTP and likelihood of disease can assist in imaging appropriate populations. Thus, a decision to select CTPA or VQ requires an assessment of feasibility, the likelihood of obtaining a diagnostic VQ scan result and PTP.

Lastly, these recommendations acknowledge that radiation exposure with diagnostic tests is evolving, and the next generation of CT scanners are likely to rival radiation exposure of VQ scanning. Clinicians should have an understanding of the diagnostic tests available in their institutions to optimally use these guidelines.

\section{Good practice statements}

For patients with suspected PE in whom diagnostic imaging is required, a baseline chest radiograph may identify an alternate diagnosis to account for the patient's symptoms and potentially avoid further diagnostic imaging. The chest radiograph can also be used to assess the likelihood of obtaining a diagnostic VQ scan result. Patients with an abnormal chest radiograph are more likely to have a nondiagnostic VQ scan, in which case CTPA is preferred over VQ scanning assuming no contraindications to CTPA.

A follow-up ultrasound for patients with lower or upper extremity DVT at the time of anticoagulant discontinuation can document any residual thrombus at end of the treatment period. This provides a baseline with which to compare any subsequent ultrasound images if patients present with suspected recurrence and undergo repeat ultrasound testing.

\section{Introduction}

\section{Aim of these guidelines and specific objectives}

The purpose of these guidelines is to provide evidence-based recommendations about the diagnostic evaluation of patients with suspected PE, DVT, and upper extremity DVT. Diagnostic pathways based on the expected prevalence of VTE are presented and guidance on testing is provided based on clinically relevant and accepted thresholds for accepting error. The recommendations are further guided by principles of minimizing radiation exposure and the required number of diagnostic tests to meet the accepted thresholds where possible. These guidelines are intended to guide clinicians in selecting optimal diagnostic tests that are more likely to result in a diagnostic result, reduce the number of diagnostic tests, and minimize exposure to radiation. The target audience includes patients, hematologists, emergency medicine physicians, general practitioners, internists, radiologists, vascular medicine specialists, other clinicians, and decision-makers. Policy makers interested in these guidelines include those involved in developing local, national or international plans with the goal to reduce the incidence of and manage VTE and evaluate direct and indirect harms and costs related to VTE. This document may also serve as the basis for adaptation by local, regional or national guideline panels.

\section{Description of the health problems}

The incidence of VTE is $\sim 1$ per 1000 persons per year. Establishing an accurate diagnosis of PE and DVT of the lower or upper extremities is critical, as a diagnosis of VTE is closely linked to administering anticoagulant treatment. Anticoagulation is an effective treatment of VTE but is associated with unnecessary risks of bleeding, financial cost and inconvenience if patients are mistakenly 
diagnosed with VTE. For patients with VTE in whom the diagnosis is missed, untreated VTE is associated with an increased risk of complications including life-threatening or fatal PE, pulmonary hypertension, thrombus extension and embolization and postthrombotic syndrome. Clinical assessment alone is unreliable in establishing or excluding a diagnosis of VTE and objective testing is required.

Contemporary diagnostic strategies for VTE are based on an assessment of the clinical probability of VTE in the population of interest prior to diagnostic testing (PTP). Classifying patients into low, intermediate, or high, or alternately unlikely and likely, clinical PTP of VTE is done using standardized clinical prediction rules. Examples of clinical prediction rules include the Wells score for DVT and PE and the Geneva score for PE. PTP alone is not sufficient to rule in or rule out VTE, so even low risk or unlikely PTP patients are required to undergo diagnostic testing. In the emergency department setting, PERC can be applied to determine whether patients are likely or unlikely to have PE and whether additional diagnostic testing with D-dimer is warranted. ${ }^{8}$ Patients with none of the criteria are considered low risk and no D-dimer or additional testing is performed. Classification of patients by PTP provides an estimate of the prevalence of VTE in that patient subgroup. Patients with low or unlikely clinical PTP have a low prevalence of VTE and those with high or likely clinical PTP have a high prevalence of VTE. The prevalence of disease in a population influences the predictive value of diagnostic tests. A diagnostic test in a population with a low prevalence of VTE has a high negative predictive value (ie, patients who test negative truly do not have VTE) but a low positive predictive value (ie, patients who test positive truly have VTE). Conversely, the same test in a population with a high prevalence of VTE has a high positive predictive value and a low negative predictive value.

Diagnostic tests themselves are associated with error. In the absence of a diagnostic test that identifies VTE with complete accuracy, VTE diagnosis has evolved to estimate PTP, ideally using a clinical prediction rule, followed by 1 or more diagnostic tests to obtain a posttest probability of VTE. In a patient population with a low clinical PTP for VTE and thus a low prevalence of disease, a diagnostic test may reduce the posttest probability of VTE below a certain threshold such that one would be confident that the diagnosis of VTE is excluded. In a population with a high clinical PTP for VTE and a high prevalence of disease, the same diagnostic test may not be sufficient for excluding VTE or specific enough to establish the diagnosis, requiring a subsequent diagnostic test to achieve the desired posttest probability threshold.

The concept of a posttest probability threshold has high utility for clinical decision-making. Because most patients investigated for suspected VTE do not have VTE, diagnostic strategies are currently validated in management studies by using the observed rate of VTE at 3 months in untreated patients. In studies using reference standards of negative venography (for DVT) or pulmonary angiography (for PE), the observed VTE rate is $\sim 2 \%{ }^{9,10}$ This $2 \%$ FN rate in the population can be considered the posttest probability threshold for an acceptable diagnostic pathway. Thus, if the posttest probability (FN rate) of VTE following a pathway of 1 or more diagnostic tests is $<2 \%$, this would safely exclude the diagnosis of VTE.

Modern diagnostic techniques have evolved such that current CT scanners are able to detect PE at a subsegmental level and ultrasound capable of detecting DVT within calf veins. The clinical relevance of such findings is controversial and potentially contributes to the concept of overdiagnosis of VTE. Anticoagulant treatment of subsegmental PE or calf vein thrombosis exposes patients to the risks of anticoagulation potentially without concomitant benefit, and studies evaluating the clinical importance and appropriate management of these findings are needed. Nonetheless, this emphasizes the necessity of imaging clinically appropriate populations, and determining the PTP prior to diagnostic testing can assist in determining whether and what diagnostic testing is appropriate.

\section{Methods}

The guideline panel developed and graded the recommendations and assessed the certainty in the supporting evidence following the GRADE approach.5,6 The overall guideline development process, including funding of the work, panel formation, management of conflicts of interest, internal and external review, and organizational approval, was guided by ASH policies and procedures derived from the GIN-McMaster Guideline Development Checklist (https:// heigrade.mcmaster.ca/home) and intended to meet recommendations for trustworthy guidelines by the National Academy of Medicine and the GIN..$^{1-4}$ An article detailing the methods used to develop these guidelines is forthcoming.

\section{Organization, panel composition, planning, and coordination}

The work of this panel was coordinated with that of 9 other guideline panels (addressing other aspects of VTE) by ASH and the McMaster GRADE Centre (funded by ASH under a paid agreement). Project oversight was provided initially by a coordination panel, which reported to the ASH Committee on Quality, and then by the coordination panel chair (Adam Cuker) and vice chair (H.J.S.). ASH vetted and appointed individuals to the guideline panel. The McMaster GRADE Centre vetted and retained researchers to conduct systematic reviews of evidence and coordinate the guideline development process, including the use of the GRADE approach. The membership of the panel and the systematic review team is described in Supplement 1.

The panel included hematologists with clinical and research expertise on the guideline topic, physicians from other disciplines with similar expertise (including radiologists, internists, and emergency medicine specialists), methodologists with expertise in evidence appraisal and guideline development, and 2 patient representatives. The panel chair was an ASH member and content expert. The vice chair was a clinician and methodologist with experience in guideline development processes and diagnostic methodology.

In addition to synthesizing evidence systematically, the McMaster GRADE Centre supported the guideline development process, including determining methods, preparing agendas and meeting materials, and facilitating panel discussions. The panel's work was done using web-based tools (www.surveymonkey.com and www.gradepro.org) and 1 face-to-face as well as online meetings.

\section{Guideline funding and management of conflicts of interest}

Development of these guidelines was wholly funded by $\mathrm{ASH}$, a nonprofit medical specialty society that represents hematologists. Most members of the guideline panel were members of ASH. ASH staff supported panel appointments and coordinated meetings but had no role in choosing the guideline questions or determining the recommendations. 
Members of the guideline panel received travel reimbursement for attendance at in-person meetings. One of the patient representatives received an honorarium of $\$ 200$ US dollars; the other declined this. The panelists received no other payments. Through the McMaster GRADE Centre, some researchers who contributed to the systematic evidence reviews received salary or grant support. Other researchers participated to fulfill requirements of an academic degree or program.

Conflicts of interest of all participants were managed according to ASH policies based on recommendations of the Institute of Medicine $^{11}$ and GIN. ${ }^{4}$ At the time of appointment, a majority of the guideline panel, including the chair and the vice chair, had no conflicts of interest as defined and judged by $\mathrm{ASH}$, that is, no current material interest in any commercial entity with a product that could be affected by the guidelines. Some panelists disclosed new interests or relationships during the development process, but the balance of the majority was maintained.

Before appointment to the panel, individuals disclosed both financial and nonfinancial interests. Members of the VTE Guideline Coordination Panel reviewed the disclosures and judged which interests were conflicts and should be managed. Supplement 2 provides the complete "Disclosure of Interests" forms of all panel members. In part $A$ of the forms, individuals disclosed material interests for 2 years prior to appointment. In part B, they disclosed interests that were not mainly financial. Part $\mathrm{C}$ summarizes $\mathrm{ASH}$ decisions about which interests were judged to be conflicts. Part D describes new interests disclosed by individuals after appointment.

$\mathrm{ASH}$ policy required that panel members with a current, direct financial interest in a commercial entity with any product that could be affected by the guidelines be recused from making judgments about relevant recommendations. ${ }^{4,12-14}$ However, no panel members met this criterion and hence no individuals were recused from making judgments about any recommendations.

None of the researchers who contributed to the systematic evidence reviews or who supported the guideline development process had any current, material interest in a commercial entity with any product that could be affected by the guidelines. Supplement 3 provides the complete disclosure of interest forms of researchers who contributed to these guidelines.

\section{Formulating specific clinical questions and determining outcomes of interest}

The panel used the GRADEpro Guideline Development Tool (www.gradepro.org) ${ }^{15}$ and SurveyMonkey (www.surveymonkey.com) to brainstorm, then prioritize the following questions addressed by these guidelines:

1. In a patient population with a low clinical probability of $P E$, what is the optimal diagnostic strategy to evaluate for suspected first episode PE?

2. In a patient population with an intermediate clinical probability of $P E$, what is the optimal diagnostic strategy to evaluate for suspected first episode PE?

3. In a patient population with a high clinical probability of PE, what is the optimal diagnostic strategy to evaluate for suspected first episode PE?

4. For patients with a prior history of PE, what is the optimal diagnostic strategy to evaluate for suspected recurrent PE?
5. In a patient population with a low clinical probability of lower extremity DVT, what is the optimal diagnostic strategy to evaluate for suspected first episode DVT?

6. In a patient population with an intermediate clinical probability of lower extremity DVT, what is the optimal diagnostic strategy to evaluate for suspected first episode DVT?

7. In a patient population with a high clinical probability of lower extremity DVT, what is the optimal diagnostic strategy to evaluate for suspected first episode DVT?

8. For patients with a prior history of DVT, what is the optimal diagnostic strategy to evaluate for suspected recurrent DVT?

9. In a patient population with an unlikely clinical probability of upper extremity DVT, what is the optimal diagnostic strategy to evaluate for suspected upper extremity DVT?

10. In a patient population with a likely clinical probability of upper extremity DVT, what is the optimal diagnostic strategy to evaluate for suspected upper extremity DVT?

Diagnosis of PE, DVT of the lower and upper extremities, and recurrent PE and DVT were prioritized. Diagnosis of thrombosis affecting other areas, for example, cerebral vein, splanchnic veins (portal vein, splenic vein, mesenteric veins), ovarian veins, retinal veins, is outside the scope of these guidelines. The diagnostic tests considered were limited to those commonly encountered in clinical practice. Although pulmonary angiography and venography have historically been used as reference standards for diagnostic tests of PE and DVT, these tests require technical expertise that is increasingly difficult to access and carry risks to patients that in the contemporary era are not acceptable. Newer diagnostic modalities including VQ SPECT and magnetic resonance imaging (MRI) are emerging but are not the standard of care in most countries. Consequently, the diagnostic tests considered in these guidelines were limited to highly sensitive D-dimer, VQ scan, multidetector CTPA (studies from 2000 forward), compression ultrasonography of the proximal leg veins or whole-leg ultrasonography (where both the proximal and distal leg veins are examined) plus or minus color Doppler (referred to as duplex ultrasonography).

PTP was assumed to be assessed using validated clinical prediction rules. A systematic review of published clinical prediction rules, comparison of different clinical prediction rules and recommendations for use of 1 clinical prediction rule over another are outside the scope of these guidelines. The clinical prediction rule used for individual studies is provided in the evidence tables. ${ }^{16}$ This guideline used a 3-level clinical prediction rule, although 2 level or simplified clinical prediction rules exist. In general, patients classified into unlikely and likely VTE categories correspond to the low and intermediate-high categories, respectively.

After the panel prioritized the questions, the chairs developed diagnostic pathways which were refined through an iterative process with input from the panel. These pathways were used to guide the evidence synthesis and recommendations. The panel selected outcomes of interest for each question a priori, following the approach described in detail elsewhere. ${ }^{17}$ In brief, the panel brainstormed all possible outcomes, then rated their relative importance for decision-making following the GRADE approach. ${ }^{18}$ During this rating process, the panel used definitions of the outcomes ("marker states") that were developed for these guidelines by the 
McMaster GRADE Centre. Rating outcomes by their relative importance can focus attention on those outcomes that are considered most important and help to resolve or clarify potential disagreements. The panel rated the following outcomes as critical for decision making across the PE diagnosis questions: mortality, PE, major bleeding, intracranial hemorrhage, and chronic thromboembolic pulmonary hypertension, in addition to the diagnostic accuracy outcomes (FP, FN, TP, and TN test results). The panel rated the following outcomes as critical for decision making across the DVT diagnosis questions: mortality, proximal DVT, distal DVT, major bleeding, and intracranial hemorrhage, in addition to the diagnostic accuracy outcomes (FP, FN, TP, TN test results). In most instances, there was no direct evidence assessing the effect of using 1 diagnostic pathway vs another on patient outcomes or directly comparing the accuracy of different diagnostic pathways. In some circumstances where the diagnostic pathway of interest was evaluated, details regarding the specific number of patients (to determine diagnostic test accuracy outcomes) was not provided or the outcomes selected differed from the prioritized outcomes from the panel. To make judgments about health care-related desirable and undesirable effects, the panel reviewed all the potential pathways and determined the downstream consequences related to a particular test result as well as the complications of testing itself. This affected the degree of certainty in the recommendations.

\section{Evidence review and development of recommendations}

For each guideline question, the McMaster GRADE Centre and the systematic review team prepared a GRADE "Evidence-to-Decision" (EtD) framework, using the GRADEpro Guideline Development Tool (www.gradepro.org). ${ }^{19-21}$ Modifications were made to the EtD framework to address diagnostic strategies and potential diagnostic pathways. The EtD table summarized the results of systematic reviews of the literature that were performed specifically for these guidelines $^{19,20}$ The EtD table addressed effects of interventions including potential benefits and harms of the diagnostic test, test accuracy, natural history of VTE, resource utilization, values and preferences (relative importance of outcomes), equity, feasibility, and acceptability. The guideline panel reviewed draft EtD tables before, during and after the guideline panel meeting and made suggestions for corrections and additional evidence. To ensure that recent studies were not missed, searches (Supplement 4) were updated until October 2017.

The systematic review team followed the general methods outlined in the Cochrane Handbook for Systematic Reviews (https://training. cochrane.org/handbook) for conducting new systematic reviews of the different diagnostic tests and strategies. Risk of bias was assessed for each test accuracy study using the QUADAS-2 tool and for other study designs using the appropriate risk of bias tool. ${ }^{22}$ In addition to conducting systematic reviews of test accuracy and studies directly assessing the effects of tests on patient outcomes, the review team searched for prevalence estimates, natural history and progression of VTE.

The panel guided decisions about the specific D-dimer test assays, cutoffs, and machines to be included in the reviews. Box 1 summarizes these decisions.

The MacGRADE Centre performed systematic reviews to synthesize published evidence about patients' values and preferences,

\section{Box 1: Summary of decisions}

Population: This guideline is not intended to address screening for VTE in asymptomatic patients, or diagnosis of VTE in pediatric patients, pregnant women, or patients with superficial vein thrombosis.

Test: This guideline evaluates quantitative high-sensitivity D-dimer assays, multidetector CTPA, VQ scanning, compression ultrasonography, and compression ultrasonography with color Doppler (duplex ultrasonography). It does not evaluate low-sensitivity or qualitative D-dimer assays (eg, SimpliRed), single-detector CT, VQ SPECT, MRI, transthoracic ultrasound, impedance plethysmography, venography, or pulmonary angiography.

costs and cost effectiveness analyses, feasibility, acceptability, and equity. Findings from all these reviews were summarized within the EtD tables. Subsequently, the certainty in the body of evidence (also known as the quality of the evidence or confidence in the estimated effects) was assessed for each of the effect estimates of the test accuracy outcomes. The evidence directly assessing the effect of tests on patient outcomes was evaluated following the GRADE approach based on the following domains: risk of bias, precision, consistency and magnitude of the estimate of effects, directness of the evidence, and risk of publication bias. The certainty was categorized into 4 levels ranging from very low to high. $6,18,23,24$

Due to the limited evidence directly assessing the effect of a test or a diagnosis pathway on patient outcomes, the systematic review team developed simple mathematical models to calculate the test accuracy of the entire diagnostic pathway, which permitted a comparison of the different pathways. The model included data obtained from the systematic reviews for VTE prevalence and the pooled diagnostic test accuracy for different tests. This method was used to explicitly present the test results while considering different prevalence estimates, multiple potential diagnostic pathways and different assumptions about the performance of the tests in different populations. The predicted overall test strategy results from the model and the quality of evidence assessed using the GRADE approach were summarized in evidence tables which allowed for a comparison of the benefits and harms of different diagnostic strategies.

The likelihood of a diagnostic VQ scan is influenced by presence of lung disease and patient age. A diagnostic result (normal or high probability) is more likely to occur in younger individuals and in those without preexisting lung disease. Diagnostic VQ scan results are less likely in older patients and those with underlying lung disease. The frequency of a nondiagnostic or indeterminate VQ scan was consequently modeled in 2 distinct populations: for patients likely to have a diagnostic VQ scan result, the prevalence of a nondiagnostic scan was estimated at $20 \%$ with $80 \%$ having a diagnostic result; for patients unlikely to have a diagnostic VQ scan result, the prevalence of a nondiagnostic scan was estimated to be $60 \%$ with $40 \%$ having a diagnostic result.

In situations where we were unable to establish population prevalence of VTE or were unable to obtain diagnostic test accuracy estimates we used the data obtained from management and accuracy studies to formulate the recommendations. 
Estimation of disease prevalence. Prevalence estimates for PE and DVT were identified from systematic reviews and metaanalyses evaluating clinical prediction rules and provided estimates of the prevalence of VTE in each clinical probability category. ${ }^{25,26}$ The most widely validated clinical prediction rules are the Wells score for PE and DVT and the Geneva score for PE, both of which have been validated in an outpatient population and evaluated in management studies. The Wells score has also been validated in an inpatient population. In determining the prevalence rates, it was noted that the reported VTE prevalence in European studies is generally higher compared with North American studies.

Prevalence of PE. Prevalence estimates for PE were obtained from a meta-analysis of 29 studies of 31215 patients where the 3 level Wells score was evaluated in 14 studies. $^{25}$ The pooled prevalence of PE in these studies was $5.7 \%$ in the low PTP category, $23.2 \%$ in the intermediate PTP, and $49.3 \%$ in the high PTP group. Prevalence estimates of 5\% (low), 20\% (intermediate), and $50 \%$ (high) were used for modeling the expected diagnostic test accuracy estimates for PE.

Prevalence of DVT. Prevalence estimates for DVT were obtained from a meta-analysis of individual patient data from 13 studies of 10002 patients using the Wells score. ${ }^{26}$ The overall prevalence of DVT was $19 \%$ with an observed prevalence for patients with a low PTP population ranging from $3.5 \%$ to $8.1 \%$, intermediate $13.3 \%$ to $23.9 \%$, and high $36.3 \%$ to $61.5 \%$. Prevalence estimates of 10\% (low), 25\% (intermediate), and $50 \%$ (high) were used for modeling the expected diagnostic test accuracy estimates for DVT.

Determination of thresholds. To rank the diagnostic pathways, threshold values for acceptable FN and FP rates were obtained through panel discussion and based on expected clinical consequences for each of these categories. A FN rate of $2 \%$ or less and a misdiagnosis (FN+FP) rate of $5 \%$ or less were established as acceptable and clinically relevant thresholds. A FN rate of $\leq 5 \%$ and a misdiagnosis rate of $\leq 10 \%$ was considered as potentially acceptable and any pathway with a $\mathrm{FN}>5 \%$ and/or a misdiagnosis rate of $>10 \%$ as unacceptable. A less conservative threshold was established for upper extremity DVT, with a FN rate of $5 \%$ or less, and a misdiagnosis rate of $10 \%$ or less. This decision was based on uncertainty in the severity of clinical outcomes associated with upper extremity DVT and an acknowledgment that the prioritized outcome of chronic thromboembolic pulmonary hypertension was unlikely to be associated with upper extremity DVT.

Recurrent VTE. Studies evaluating recurrent VTE that could provide diagnostic test accuracy estimates were searched to permit modeling of the diagnostic test characteristics in a population of patients with suspected recurrent VTE. Ultimately, the panel concluded that modeling would not be feasible given that: (1) diagnostic tests perform differently for patients with prior VTE and (2) prevalence estimates for recurrent DVT and recurrent PE were not available. Patients with prior VTE are a heterogeneous group, with absence of ongoing anticoagulation at the time of presentation being one of the strongest predictors of recurrence. Other factors that are recognized to influence recurrence include sex, the nature of the initial VTE event (provoked vs unprovoked), and presence of ongoing risk factors (eg, cancer). The studies evaluating diagnostic strategies for recurrent VTE did not clearly separate patients receiving, or not receiving, anticoagulation and the performance of the diagnostic test in each of these groups. This is particularly relevant for D-dimer testing, where the effect of anticoagulation on $D$-dimer test results is uncertain at best.

For patients with prior VTE, $30 \%$ to $50 \%$ will have residual abnormalities on ultrasound or VQ scan upon completion of anticoagulant treatment. ${ }^{27}$ Patients with a normal CTPA at the end of treatment may still have residual defects on VQ scan which are slower to resolve. Diagnostic criteria have been used to distinguish recurrent, compared with residual, VTE. For patients with suspected recurrent DVT, thrombus in a previously unaffected venous segment or an increase in venous diameter during compression of $>4 \mathrm{~mm}$ in a region with prior thrombus would be considered diagnostic for recurrent DVT. For patients with suspected recurrent $P E$, a new ventilation-perfusion defect on VQ scan or new thrombus in the pulmonary arteries that was not previously noted on CTPA would be considered diagnostic for recurrent PE. These criteria require that previous imaging results be available for comparison, a significant limitation in the diagnosis of recurrent VTE. The studies evaluating diagnostic strategies for recurrent VTE were not able to provide this level of detail in order to determine test accuracy characteristics. The panel agreed that using the test characteristics from the first episode VTE population and extrapolating to the recurrent VTE population would be inaccurate.

Establishing the prevalence of recurrent VTE was a limitation. The clinical prediction rules used to classify patients into low, intermediate and high PTP have not been validated for patients with suspected recurrence. A history of prior VTE would result in a score of at least 1 or 1.5 using the Wells score and it is likely that many patients would have at least 1 other criterion which would result in a moderate or high PTP for VTE. The prevalence reported in the specific studies evaluating diagnostic strategies for recurrent VTE was highly variable. Taken together, the panel agreed that summarizing the test performance of diagnostic tests in published management studies would be more accurate than the modeling done for the first episode VTE questions.

During a 2-day in-person meeting, the panel developed recommendations based on the evidence summarized in the EtD tables. For each recommendation, the panel took a population perspective and agreed on the following: the certainty in the evidence, the health care-related desirable and undesirable effects of the compared diagnostic strategy options and the assumptions about the values and preferences associated with the decision. The guideline panel also took into account the extent of resource use associated with alternative diagnostic options. The guideline panel agreed on the recommendations (including direction and strength), remarks, and qualifications based on the balance of all desirable and undesirable consequences. ${ }^{19,20}$ The draft EtD frameworks and recommendations were reviewed by all members of the panel. The final guidelines were reviewed and approved by the panelists listed as authors.

\section{Interpretation of strong and conditional recommendations}

The recommendations are labeled as either "strong" or "conditional" according to the GRADE approach. The words "the guideline panel recommends" are used for strong recommendations, and 


\begin{tabular}{|c|c|c|}
\hline Implications for & Strong recommendation & Conditional recommendation \\
\hline Patients & $\begin{array}{l}\text { Most individuals in this situation would want the recommended } \\
\text { course of action, and only a small proportion would not. }\end{array}$ & $\begin{array}{l}\text { The majority of individuals in this situation would want the } \\
\text { suggested course of action, but many would not. Decision aids } \\
\text { may be useful in helping patients to make decisions consistent } \\
\text { with their individual risks, values, and preferences. }\end{array}$ \\
\hline Clinicians & $\begin{array}{l}\text { Most individuals should receive the intervention. Formal decision } \\
\text { aids are not likely to be needed to help individual patients make } \\
\text { decisions consistent with their values and preferences. }\end{array}$ & $\begin{array}{l}\text { Different choices will be appropriate for individual patients, and } \\
\text { clinicians must help each patient arrive at a management } \\
\text { decision consistent with the patient's values and preferences. } \\
\text { Decision aids may be useful in helping individuals to make } \\
\text { decisions consistent with their values and preferences. }\end{array}$ \\
\hline Policy makers & $\begin{array}{l}\text { The recommendation can be adopted as policy in most situations. } \\
\text { Adherence to this recommendation according to the guideline } \\
\text { could be used as a quality criterion or performance indicator. }\end{array}$ & $\begin{array}{l}\text { Policymaking will require substantial debate and involvement of } \\
\text { various stakeholders. Performance measures should assess if } \\
\text { decision-making is appropriate. }\end{array}$ \\
\hline Researchers & $\begin{array}{l}\text { The recommendation is supported by credible research or other } \\
\text { convincing judgments that make additional research unlikely to } \\
\text { alter the recommendation. On occasion, a strong } \\
\text { recommendation is based on low or very low certainty in the } \\
\text { evidence. In such instances, further research may provide } \\
\text { important information that alters the recommendations. }\end{array}$ & $\begin{array}{l}\text { The recommendation is likely to be strengthened (for future } \\
\text { updates or adaptation) by additional research. An evaluation of } \\
\text { the conditions and criteria (and the related judgments, research } \\
\text { evidence, and additional considerations) that determined the } \\
\text { conditional (rather than strong) recommendation will help } \\
\text { identify possible research gaps. }\end{array}$ \\
\hline
\end{tabular}

"the guideline panel suggests" for conditional recommendations. Table 1 provides the suggested interpretation of strong and conditional recommendations by patients, clinicians, and health care policy makers.

\section{Document review}

Draft recommendations were reviewed by all members of the panel, revised, and then made available online on 5 December 2017, for external review by stakeholders including allied organizations, other medical professionals, patients, and the public. Eight individuals or organizations submitted comments. The document was revised to address pertinent comments, but no changes were made to recommendations. On 30 July 2018 , the ASH Guideline Oversight Subcommittee and the ASH Committee on Quality approved that the defined guideline development process was followed, and on 3 August 2018, the officers of the ASH Executive Committee approved submission of the guidelines for publication under the imprimatur of $\mathrm{ASH}$. The guidelines were then subjected to peer review by Blood Advances.

\section{How to use these guidelines}

ASH guidelines are primarily intended to help clinicians make decisions about diagnostic and treatment alternatives. Other purposes are to inform policy, education, and advocacy and to state future research needs. They may also be used by patients. These guidelines are not intended to serve or be construed as a standard of care. Clinicians must make decisions on the basis of the clinical presentation of each individual patient, ideally through a shared process that considers the patient's values and preferences with respect to the anticipated outcomes of the chosen option. Decisions may be constrained by the realities of a specific clinical setting and local resources, including but not limited to institutional policies, time limitations, and availability of treatments. These guidelines may not include all appropriate methods of care for the clinical scenarios described. As science advances and new evidence becomes available, recommendations may become outdated. Following these guidelines cannot guarantee successful outcomes. ASH does not warrant or guarantee any products described in these guidelines.

Statements about the underlying values and preferences as well as qualifying remarks accompanying each recommendation are its integral parts and serve to facilitate more accurate interpretation. They should never be omitted when recommendations from these guidelines are quoted or translated. Implementation of the guidelines will be facilitated by the related interactive forthcoming decision aids. The use of these guidelines is also facilitated by the links to the EtD frameworks and interactive summary-of-findings tables in each section.

These guideline recommendations are based on an assessment of the evidence from clinical studies evaluating diagnostic testing and management strategies at a population level. However, there are different considerations to be made when assessing an individual patient presenting with suspected VTE. The presence of individual patient comorbidities and the presence of VTE and bleeding risk factors are important considerations when selecting diagnostic tests and for decisions regarding anticoagulant use. Other factors that contribute to variability in clinical practice and are not necessarily captured in guidelines include the clinical setting where the patient is assessed, the availability of diagnostic tests at an institution, the variation in laboratory assays and imaging techniques, and geographic location. Guidelines cannot account for the variation that exists at an individual patient level that might result in a clinical judgment that differs from guideline recommendations. Ultimately, clinicians must use clinical judgment alongside guideline recommendations in determining the optimal diagnostic approach.

\section{Recommendations}

\section{Diagnosis of PE}

The panel judged the ranking of pathways based on predetermined thresholds of acceptable FN and misdiagnosis (FN+FP) rates for diagnosis of $\mathrm{PE}$. Pathways that provided the best balance of desirable and undesirable effects remained at or below a threshold of $20 \mathrm{FN}$ results per 1000 patients tested 
Table 2. Summary of results of studies informing sensitivity and specificity of tests for diagnosis of PE

\begin{tabular}{|c|c|c|c|c|}
\hline Test & No. of participants (studies) & Sensitivity (95\% Cl) & Specificity $(95 \% \mathrm{Cl})$ & Quality of evidence \\
\hline CTPA & 3929 (15) & $0.93(0.88-0.96)$ & $0.98(0.96-0.99)$ & Moderate ${ }^{\star}, t, \neq$ \\
\hline D-dimer & $20568(30)$ & $0.97(0.96-0.98)$ & $0.39(0.36-0.43)$ & Moderate ${ }^{\star}, t, \S$ \\
\hline Age-adjusted D-dimer & $2885(1)$ & $0.99(0.98-1.00)$ & $0.47(0.45-0.49)$ & High\| \\
\hline Proximal ultrasound & $1715(7)$ & $0.49(0.31-0.66)$ & $0.96(0.95-0.98)$ & Low $^{\star}, \boldsymbol{q}, \#$ \\
\hline VQ 1 & 3994 (13) & $0.58(0.50-0.66)$ & $0.98(0.96-0.99)$ & Moderate $^{\star}, t,{ }^{\star \star}$ \\
\hline VQ 2 & 3 994 (13) & $0.98(0.95-0.99)$ & $0.36(0.27-0.45)$ & Moderate ${ }^{*}, t,+\dagger$ \\
\hline VQ 3 & 3994 (13) & $0.96(0.91-0.98)$ & $0.95(0.89-0.98)$ & $\mathrm{High}^{\star}, t, \neq, \neq$ \\
\hline
\end{tabular}

$\mathrm{Cl}$, confidence interval; VQ 1, high probability scan interpreted as positive, low/nondiagnostic/normal scan interpreted as negative; VQ 2, high/low/nondiagnostic probability scan

interpreted as positive, normal scan interpreted as negative; VQ 3 , high probability scan interpreted as positive, normal scan interpreted as negative.

${ }^{*}$ Certainty in evidence not downgraded for risk of bias, although few studies had a combination of reference standards that were judged to be acceptable by a panel of clinical experts.

tCertainty in evidence was downgraded for indirectness in instances where this test was not the index test in a diagnostic pathway. There was a lack of data on the accuracy of this test

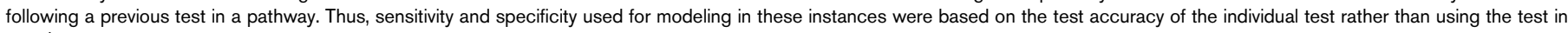
a pathway.

₹Certainty in evidence was downgraded for serious unexplained inconsistency in sensitivity, with a range from $63 \%$ to $99.2 \%$. Minor inconsistency for specificity noted but judged to be insufficient to downgrade the certainty in evidence.

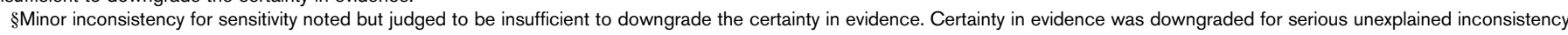
in specificity, with a range from $12.8 \%$ to $64 \%$.

$\|$ Certainty in evidence not downgraded for imprecision given the large population size, although only 1 prospective age-adjusted D-dimer study was identified for analysis.

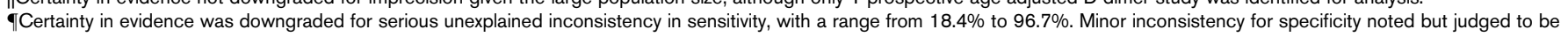
insufficient to downgrade the certainty in evidence.

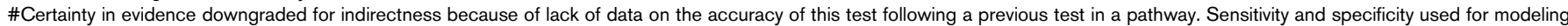
are based on the test accuracy of the individual test rather than using the test in a pathway.

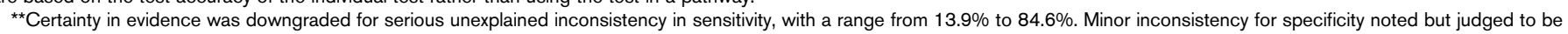
insufficient to downgrade the certainty in evidence.

t+Minor inconsistency for sensitivity noted but judged to be insufficient to downgrade the certainty in evidence. Certainty in evidence was downgraded for serious unexplained inconsistency in specificity, with a range from $10.9 \%$ to $81.8 \%$.

\#\#Minor inconsistency for sensitivity and specificity noted but judged to be insufficient to downgrade the certainty in evidence.

$(\leq 2 \%)$ and a threshold of 50 misdiagnosed results per 1000 patients tested (FN+FP $\leq 5 \%)$.

Summary of evidence. We included a total of 53 studies and 28969 patients to inform estimates of test accuracy (Table 2). Additionally, we included 22 studies and 15865 patients to inform estimates of effects of performing a test on patient important outcomes for patients suspected of having PE. The references for the included studies are as follows: CTPA, ${ }^{28-42}$ D-dimer, ${ }^{30,31,36,38-40,43-66}$ age-adjusted D-dimer, ${ }^{40}$ proximal compression ultrasound, ${ }^{39,67-72} \mathrm{VQ}^{28,30,34,68,71,73-80}$

\section{Low PTP/prevalence ( $\leq 5 \%)$}

Question: In a patient population with a low clinical probability of $P E$, what is the optimal diagnostic strategy to evaluate for suspected first episode PE?

\section{Recommendation 1a}

The ASH guideline panel recommends using a strategy starting with D-dimer for excluding $\mathrm{PE}$ in a population with low prevalence/PTP $(\leq 5 \%)$, followed by VQ scan or CTPA for patients requiring additional testing. If $\mathrm{D}$-dimer is not readily available, alternate acceptable strategies include performing VQ scan or CTPA alone. (Strong recommendation for D-dimer based on high certainty in the evidence of effects on clinical outcomes $\oplus \oplus \oplus \oplus$ and moderate certainty in the evidence of diagnostic accuracy studies $\oplus \oplus \oplus \mathrm{O}$; conditional recommendation for VQ scan or CTPA based on very low certainty in the evidence of effects on clinical outcomes $\oplus 000$ and low certainty in the evidence from diagnostic accuracy studies $\oplus \oplus \bigcirc)$ ).

\section{Remarks:}

- For patients presenting to an emergency department with a low probability of PE, PERC may be used to determine whether D-dimer testing is warranted.

- Validated clinical decision rules were used to assess clinical probability of $\mathrm{PE}$ in studies evaluating different diagnostic strategies for patients suspected of having a first episode PE. The Geneva score has been validated only in an outpatient population. If a 2-level clinical decision rule is used, this recommendation corresponds to the "unlikely PE" category. A decision to start with D-dimer assumes the results will be obtained in a timely manner and that the cost of D-dimer screening is offset by avoiding unnecessary VQ or CTPA for patients at low PTP for PE. If the D-dimer strategy is followed, a highly sensitive D-dimer assay is required. A negative D-dimer rules out $\mathrm{PE}$ and no additional testing or anticoagulation is required. D-dimer has limited utility in hospitalized patients and in certain patient populations (postsurgical, pregnant) due to the high frequency of positive D-dimer results with standard thresholds.

- Use of an age-adjusted D-dimer cutoff in outpatients older than 50 years is as safe as the standard cutoff and increases the diagnostic utility of the test. Age-adjusted cutoff $=$ age (years) $\times 10 \mu \mathrm{g} / \mathrm{L}$ (using D-dimer assays with a cutoff of $500 \mu \mathrm{g} / \mathrm{L})$.

- VQ scan is preferred over CTPA as the subsequent test to limit radiation exposure for patients likely to have a diagnostic scan, and in centers where VQ scans are 
available with expertise to interpret the results in a timely manner. CTPA is preferred when VQ scan is not feasible.

- The strategy assumes that test results are obtained under optimal conditions. Suboptimal D-dimer or CTPA results may require repeat testing or an alternate strategy. If the VQ scan is nondiagnostic, additional testing with proximal ultrasound of the lower extremities or CTPA should be considered.

\section{Recommendation 1b}

The ASH guideline panel recommends against using a positive $\mathrm{D}$-dimer alone to diagnose $\mathrm{PE}$, and against additional testing following negative CTPA or normal VQ scan in a population with low prevalence/PTP $(\leq 5 \%)$. (See grading for recommendation 1a.)

Figure 1 presents a flowchart of recommendations $1 \mathrm{a}$ and $\mathrm{b}$.

Benefits, harms, and burden. For a population with low clinical probability of $\mathrm{PE}$, threshold criteria were met by pathways starting with D-dimer where patients with a positive D-dimer subsequently underwent testing with CTPA or VQ scan, then patients with nondiagnostic VQ scans received proximal ultrasound or CTPA. Pathways with no follow-up testing for positive D-dimer patients resulted in a large number of FP results. CTPA used as the sole diagnostic tool was adequate for meeting these threshold criteria. The addition of subsequent tests including D-dimer, compression ultrasound, or VQ after CTPA increased the FP rate beyond acceptable thresholds. Pathways starting with VQ scan met threshold criteria if nondiagnostic scans were followed by additional testing (CTPA or compression ultrasound). The EtD framework is online at: https://dbep.gradepro.org/profile/ e9600faf-99bc-4ade-9f2f-70bf6e078f9e.

\section{Intermediate PTP/prevalence ( 20\%)}

Question: In a patient population with an intermediate clinical probability of $P E$, what is the optimal diagnostic strategy to evaluate for suspected first episode PE?

\section{Recommendation $\mathbf{2 a}$}

The ASH guideline panel suggests using a strategy starting with D-dimer for excluding PE in a population with intermediate prevalence/PTP ( 20\%), followed by VQ scan or CTPA for patients requiring additional testing. If $\mathrm{D}$-dimer is not readily available, alternate acceptable strategies include performing VQ scan or CTPA alone. Patients who are likely to have a nondiagnostic VQ scan should undergo CTPA. (Conditional recommendation for Ddimer based on high certainty in the evidence of effects on clinical outcomes $\oplus \oplus \oplus \oplus$ and moderate certainty in the evidence about diagnostic accuracy studies $\oplus \oplus \oplus \mathrm{O}$; conditional recommendation for VQ scan or CTPA based on very low certainty in the evidence of effects on clinical outcomes $\oplus 000$ and moderate certainty in the evidence from diagnostic accuracy studies $\oplus \oplus \oplus \bigcirc)$.

\section{Remarks:}

- Validated clinical decision rules were used to assess clinical probability of PE in studies evaluating different diagnostic strategies for patients suspected of having a first episode PE. The Geneva score has been validated only in an outpatient population.

- A decision to start with D-dimer assumes the results will be obtained in a timely manner and that the cost of D-dimer screening is offset by avoiding unnecessary VQ and CTPA for patients at intermediate PTP for PE. If the D-dimer strategy is followed, a highly sensitive D-dimer assay is required. A negative D-dimer rules out PE and no additional testing or anticoagulation is required. D-dimer has limited utility in hospitalized patients and in certain patient populations (postsurgical, pregnant) due to the high frequency of positive D-dimer results with standard thresholds.

- Use of an age-adjusted D-dimer cutoff in outpatients older than 50 years is as safe as the standard cutoff and increases the diagnostic utility of the test. Age-adjusted cutoff $=$ age (years) $\times 10 \mu \mathrm{g} / \mathrm{L}$ (using D-dimer assays with a cutoff of $500 \mu \mathrm{g} / \mathrm{L})$.

- VQ scan is preferred over CTPA as the subsequent test to limit radiation exposure for patients likely to have a diagnostic scan, and in centers where VQ scans are available with expertise to interpret the results in a timely manner. CTPA is preferred when VQ scan is not feasible.

- The strategy assumes that test results are obtained under optimal conditions. Suboptimal D-dimer or CTPA results may require repeat testing or an alternate strategy. If the $\mathrm{VQ}$ scan is nondiagnostic, additional testing with CTPA should be considered.

\section{Recommendation $\mathbf{2 b}$}

The ASH guideline panel recommends against using a positive D-dimer alone to diagnose PE, and against additional testing following negative CTPA or normal VQ scan in a population with intermediate prevalence/PTP ( 20\%). (See grading for recommendation 2a.)

Figure 2 presents a flowchart of recommendations $2 a$ and $b$.

Benefits, harms, and burden. For a population with intermediate clinical probability of PE, threshold criteria were met by pathways starting with D-dimer where patients with a positive D-dimer subsequently underwent testing by CTPA or VQ scan, then patients with nondiagnostic VQ scans received CTPA. Pathways with no follow-up testing for positive D-dimer patients resulted in a large number of FP results. CTPA used as the sole diagnostic tool was adequate for meeting these threshold criteria. The addition of subsequent tests including D-dimer, proximal ultrasound, or VQ scan after CTPA increased the FP rate beyond acceptable criteria. Pathways starting with VQ scan met threshold criteria if nondiagnostic scans were 


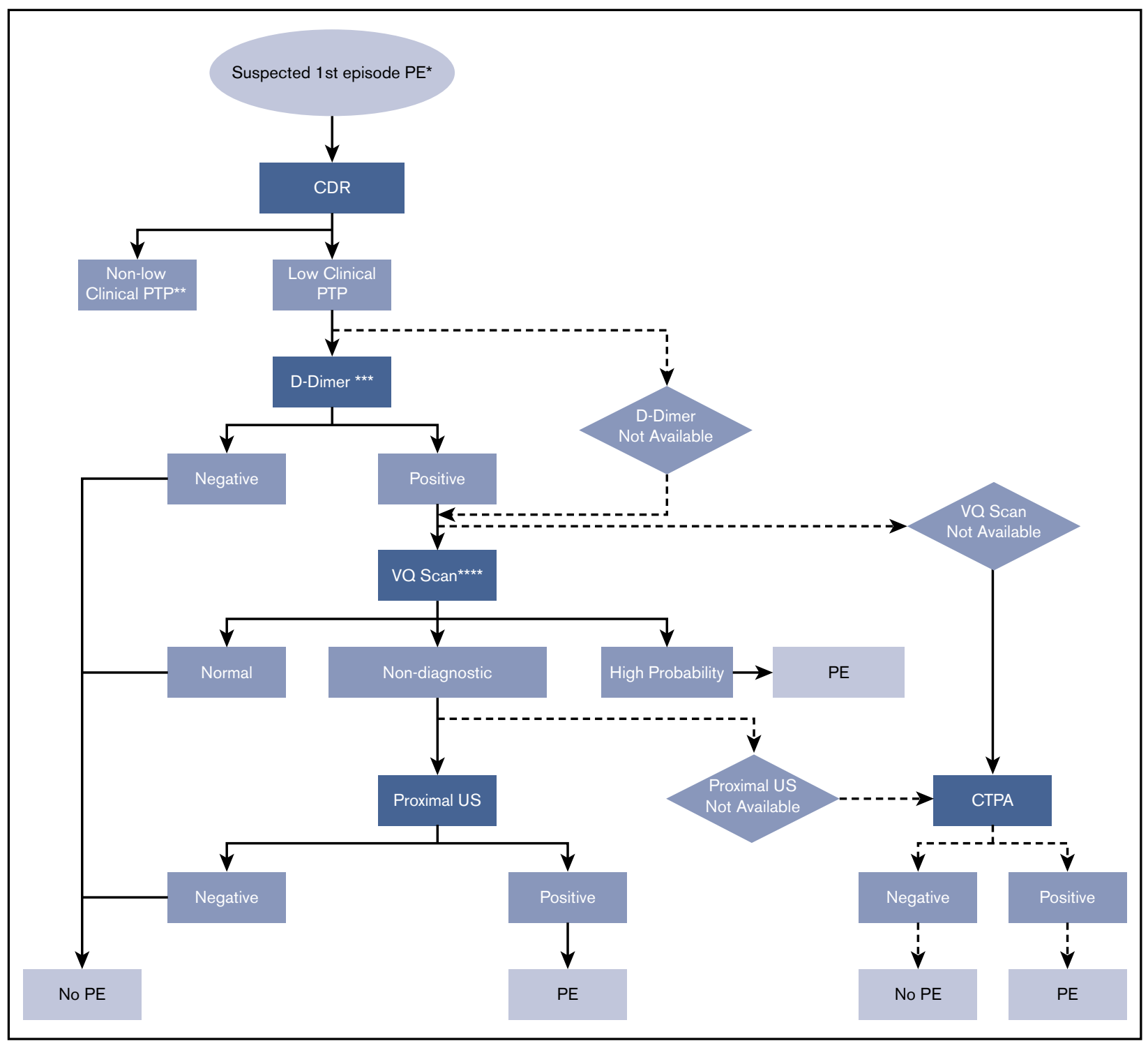

Figure 1. Flowchart for recommendations $1 \mathbf{a}$ and $\mathbf{b}$ (diagnosis of PE for patients with low PTP/prevalence [ $\leq 5 \%]$ ). *Hemodynamically stable, nonpregnant patient. ${ }^{\star \star}$ See other algorithms. ${ }^{\star \star \star}$ Highly sensitive D-dimer. ${ }^{\star \star \star \star} \mid$ f feasible. CDR, clinical decision rule; US, ultrasound.

followed by CTPA. The EtD framework is shown at: https://dbep. gradepro.org/profile/2d575b1d-8e36-43db-8805-713732e1508a.

\section{High PTP/prevalence ( $(50 \%)$}

Question: In a patient population with a high clinical probability of $P E$, what is the optimal diagnostic strategy to evaluate for suspected first episode PE?

\section{Recommendation 3a}

The ASH guideline panel suggests using a strategy starting with CTPA for assessing patients suspected of having PE in a population with high prevalence/PTP $(\geq 50 \%)$. (Conditional recommendation for CTPA based on very low certainty in the evidence of effects on clinical outcomes $\oplus 00 \bigcirc$ and moderate certainty in the evidence of diagnostic accuracy studies $\oplus \oplus \oplus \bigcirc)$.

\section{Remarks:}

- Validated clinical decision rules were used to assess clinical probability of PE in studies evaluating different diagnostic strategies for patients suspected of having a first episode PE. The Geneva score has been validated only in an outpatient population. If a 2-level clinical decision rule is used, this recommendation corresponds to the "likely PE" category.

- The strategy assumes that test results are obtained under optimal conditions. Suboptimal CTPA results may require repeat testing.

- If CTPA is not feasible (eg, contrast dye allergy, renal disease, unavailability), VQ scan may be 


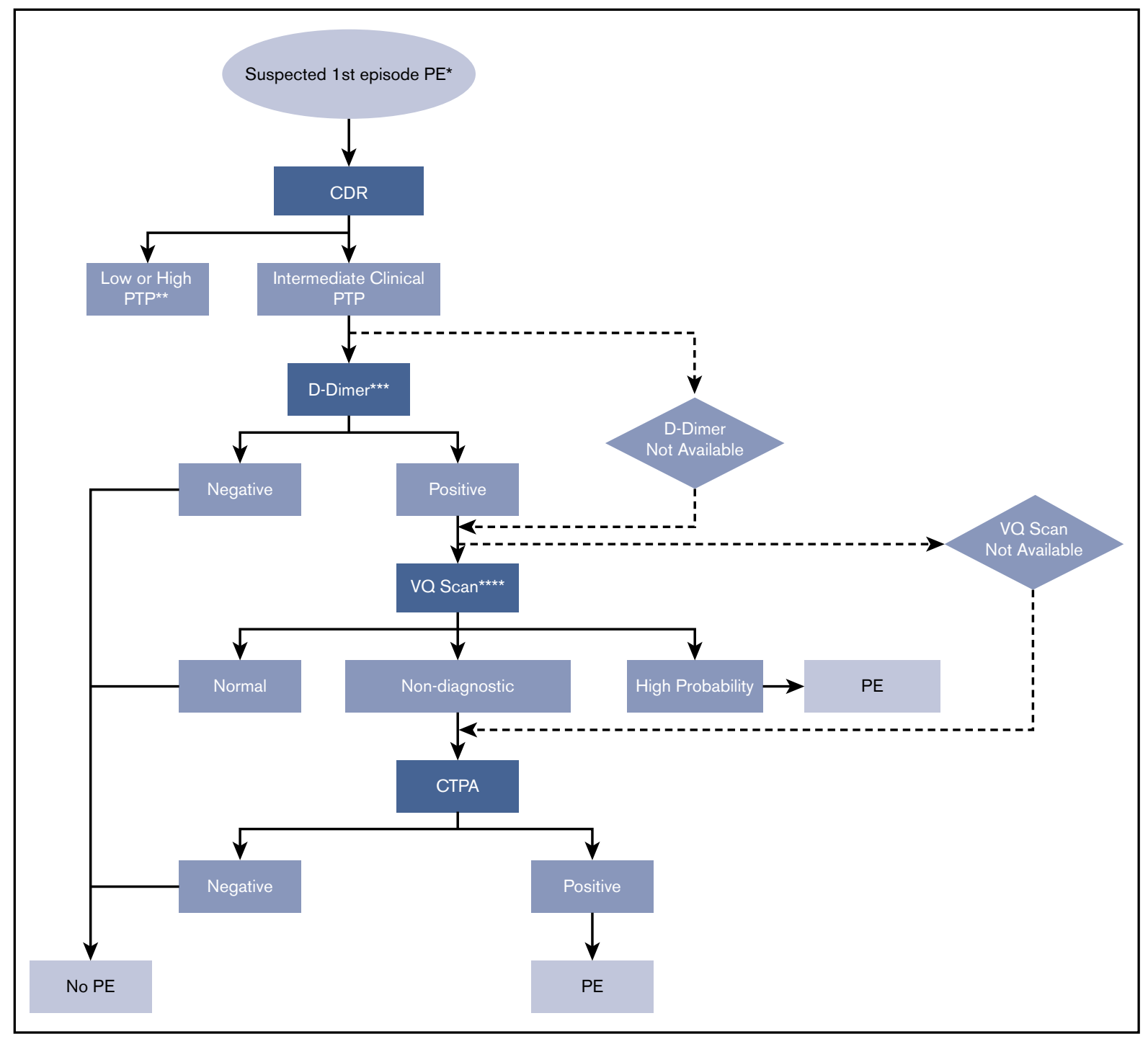

Figure 2. Flowchart for recommendations $2 a$ and $\mathbf{b}$ (diagnosis of PE for patients with intermediate PTP/prevalence [ 20\%]). *Hemodynamically stable, nonpregnant patient. ${ }^{* *}$ See other algorithms. ${ }^{* *}$ Highly sensitive D-dimer. ${ }^{* \star * \star} \mid f$ feasible.

acceptable if nondiagnostic scans are followed by additional testing.

- In cases where clinical suspicion for PE remains high with a negative initial CTPA, additional testing with VQ scan or proximal ultrasound of the lower extremities may be considered.

\section{Recommendation $\mathbf{3 b}$}

The ASH guideline panel recommends against using a positive D-dimer alone to diagnose PE, and against using D-dimer as a subsequent test following a negative CT scan in a population with high prevalence/PTP ( $\geq 50 \%$ ). (See grading for recommendation 3a.)
Figure 3 presents a flowchart of recommendations $3 a$ and $b$.

Benefits, harms, and burden. For a population with high clinical probability of PE, a negative CTPA alone did not meet the threshold criteria. The addition of subsequent tests including D-dimer, proximal ultrasound, or VQ scan after CTPA met threshold criteria. Specifically, after negative CTPA, additional testing with proximal ultrasound, positive D-dimer followed by proximal ultrasound or VQ scan where nondiagnostic scans were followed by proximal ultrasound met threshold criteria for acceptability. Possibly acceptable pathways included VQ scan where nondiagnostic scans were followed by CTPA, and Ddimer where positive results were followed by CTPA or VO scan. The panel discussed the utility of performing additional testing following a negative CTPA. The diagnostic test accuracy studies included in the systematic review did not specify the level of the PE diagnosed (eg, mainstem, lobar, segmental, or subsegmental) and estimates reflect a combination of $P E$ at all levels. The panel 


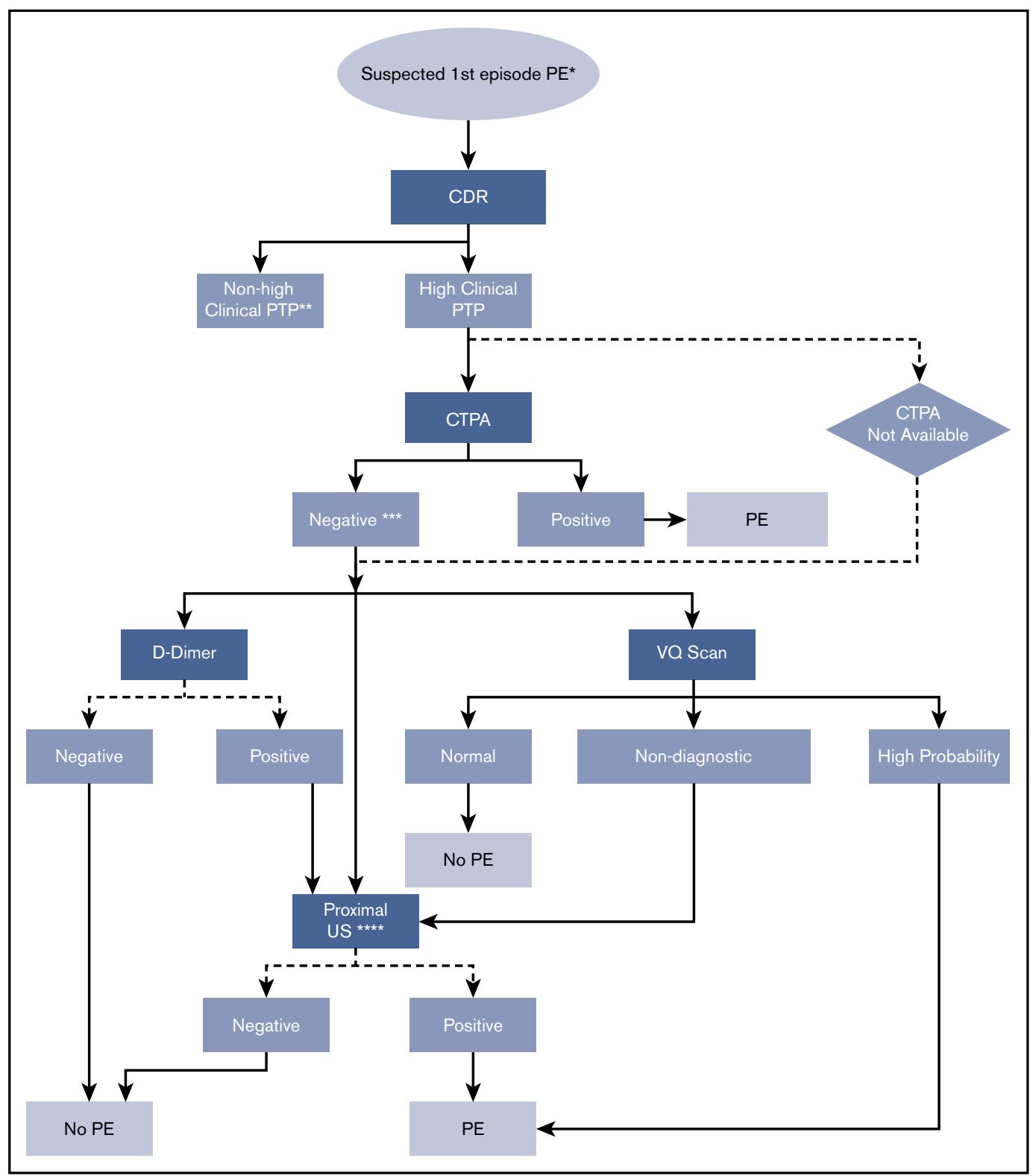

Figure 3. Flowchart for recommendations 3a and $\mathbf{b}$ (diagnosis of PE for patients with high PTP/prevalence [ $\geq \mathbf{5 0} \%]$ ). *Hemodynamically stable, nonpregnant patient. ${ }^{* *}$ See other algorithms. ${ }^{* * *}$ In selected situations, negative CTPA may be sufficient to rule out PE. ${ }^{* * *}$ Serial proximal US if clinical PTP $>50 \%$.

felt that the rate of FN scans for clinically significant PE was low, and that additional testing was unlikely to be of value. However, the panel acknowledged there may be clinical circumstances where additional testing following a negative CTPA may be considered based on clinical judgment. Pathways starting with VQ scan where nondiagnostic results were followed by proximal ultrasound, and any pathway with no follow-up testing for positive D-dimer patients resulted in a large number of positive results. The EtD framework is shown at: https://dbep.gradepro.org/profile/6affa6fc-0c1c-44e0a901-2558ee36032b.

\section{Recurrent PE}

Question: For patients with a prior history of PE, what is the optimal diagnostic strategy to evaluate for suspected recurrent PE?

\section{Recommendation 4}

The ASH guideline panel suggests using a strategy starting with D-dimer for excluding recurrent PE in a population with unlikely PTP. Patients with a positive D-dimer or those who have a likely PTP should undergo CTPA. (Conditional recommendation for Ddimer and CTPA based on low certainty in the evidence of effects on clinical outcomes $\oplus \oplus \bigcirc \bigcirc$ and moderate certainty in the evidence from diagnostic accuracy studies $\oplus \oplus \oplus \mathrm{)})$.

\section{Remarks:}

- In studies assessing this diagnostic strategy for patients suspected of having recurrent PE, Wells and Geneva 


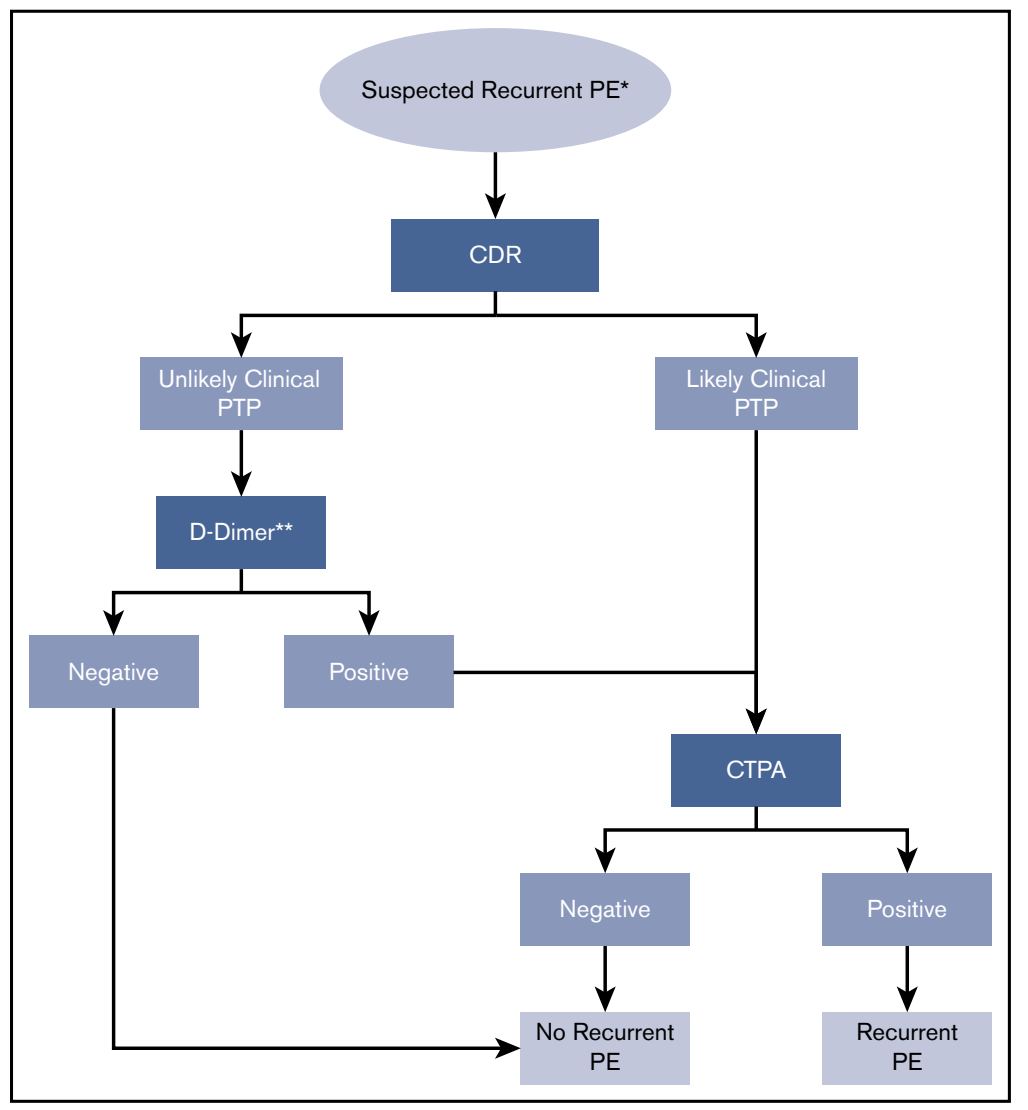

Figure 4. Flowchart for recommendation 4 (diagnosis of recurrent PE). *Hemodynamically stable, nonpregnant patient. **Highly sensitive D-dimer.

scores were used to assess clinical probability of recurrent PE. Previous VTE is a predictor in these scores, but the Wells and Geneva scores have not been specifically validated for patients with suspected recurrent PE.

- A decision to start with D-dimer assumes the results will be obtained in a timely manner and that the cost of Ddimer screening is offset by avoiding unnecessary VQ or CTPA for patients at unlikely PTP for recurrent PE. If the $\mathrm{D}$-dimer strategy is followed, a highly sensitive D-dimer assay is required. A negative D-dimer rules out PE and no additional testing or anticoagulation is required. Ddimer has limited utility in hospitalized patients and in certain patient populations (postsurgical, pregnancy) due to the high frequency of positive D-dimer results with standard thresholds. There are limited data on the utility of D-dimer for patients receiving anticoagulant therapy who present with suspected recurrent PE.

- The strategy assumes that test results are obtained under optimal conditions. Suboptimal CTPA results may require repeat testing.

- If prior imaging is available, comparison of the previous and current imaging is warranted to determine whether the findings are new and represent recurrent PE.

Figure 4 presents a flowchart of recommendation 4 .

Summary of evidence. We included a total of 4 studies and 1296 patients to inform the optimal diagnostic algorithm for patients suspected of recurrent PE (Table 3 ). The references for

Table 3. Summary of results of studies informing sensitivity and specificity of tests for diagnosis of recurrent PE

\begin{tabular}{|c|c|c|c|c|}
\hline Test & No. of participants (studies) & Sensitivity $(95 \% \mathrm{Cl})$ & Specificity $(95 \% \mathrm{Cl})$ & Quality of evidence \\
\hline $\begin{array}{l}\text { D-dimer for low PTP, CTPA for low PTP } \\
\text { with positive D-dimer and high PTP }\end{array}$ & 992 (3) & $0.97(0.94-0.98)$ & $1.00(0.99-1.00)$ & Moderate* $^{*}$ \\
\hline D-dimer & $304(1)$ & $1.00(0.97-1.00)$ & $0.27(0.21-0.34)$ & Lowt,‡ \\
\hline
\end{tabular}

${ }^{*}$ Certainty in evidence was downgraded for imprecision given the small population size from the 3 recurrent PE studies identified for analysis.

tCertainty in evidence was downgraded for imprecision given the small population size from the 1 recurrent PE study identified for analysis.

¥Certainty in evidence was downgraded for risk of bias due to a secondary analysis of 2 prospective multicenter studies with a mixed population of recurrent and firsttime PE patients. 
Table 4. Imaging considerations for VQ scan and CTPA in suspected PE

\begin{tabular}{lcc}
\hline Clinical criteria or concern & V/Q & CTPA \\
\hline At risk for reaction to contrast media requiring premedication & + & $-{ }^{*}$ \\
\hline Concern over radiation to breast tissue in women & + & - \\
Renal insufficiency & + & - \\
$\begin{array}{l}\text { Suspected VTE recurrence or treatment failure with index } \\
\text { PE diagnosed by VQ scan }\end{array}$ & + & - \\
$\begin{array}{l}\text { Suspected VTE recurrence or treatment failure with index } \\
\text { PE diagnosed by CTPA }\end{array}$ & + & $+/-$ \\
$\begin{array}{l}\text { Concern over radiation to the fetus, especially first trimester } \\
\text { Minimizing the risk of missed VTE on initial imaging detected } \\
\text { at } 3 \text { mo }\end{array}$ & $+/-$ & $+/-$ \\
\hline $\begin{array}{l}\text { Timely result required and both modalities accessible } \\
\text { Alternative or concomitant diagnosis actively sought } \\
\text { (eg, malignancy, pneumonia) }\end{array}$ & $+/-$ \\
\hline $\begin{array}{l}\text { Abnormalities present on plain radiography (eg, hyperinflation, } \\
\text { pleural effusion or other) }\end{array}$ & - & + \\
\begin{tabular}{l} 
Limited institutional access or expertise in nuclear medicine \\
\hline
\end{tabular} & - & + \\
\hline
\end{tabular}

+ , preferred; - , suboptimal; $+/-$, roughly equivalent.

*Various preparatory regimens exist to dampen what would be anticipated as a mild or moderate allergic reaction but result in imaging delays.

the included studies are as follows: D-dimer for low PTP, CTPA for low PTP with positive D-dimer and high PTP, ${ }^{16,81,82}$ D-dimer. ${ }^{83}$

Benefits, harms, and burden. For a population with suspected recurrent PE, no modeling of outcomes was performed and recommendations are based on diagnostic pathways used in management studies. The EtD framework is shown at: https://dbep.gradepro.org/profile/dc56e5e0-1329450a-882f-b7c96b1572ef.

Other EtD criteria and considerations for PE recommendations. Table 4 presents imaging considerations for VQ scan and CTPA for patients with suspected PE.

Conclusions and research needs for PE recommendations. The guideline panel determined that for D-dimer, there is high certainty evidence for using D-dimer to exclude PE for patients with low PTP/prevalence, moderate certainty evidence for patients with intermediate PTP/prevalence and very low/low evidence for patients with suspected recurrence. CTPA can be used as the only test in low and intermediate PTP/prevalence populations. In a high PTP/prevalence population, CTPA is recommended. Although additional testing following a negative CTPA improved acceptable FN and misdiagnosis rates in our modeling, the panel felt that the FN rate for clinically significant $P E$ was low and that routine additional testing following a negative CTPA should only be considered on an individual basis. Patients with nondiagnostic VQ scan results require additional testing, hence selecting patients likely to have a nondiagnostic VQ scan to undergo CTPA is appropriate. There is a need to validate clinical prediction rules for patients with recurrent PE. The clinical relevance of CTPA findings of subsegmental $P E$ are uncertain and research into optimal management is needed. Newer diagnostic modalities for PE diagnosis, including VQ SPECT, SPECT CT, and MRI require further validation in management studies.

\section{Diagnosis of lower extremity DVT}

The panel judged the ranking of pathways based on predetermined thresholds of acceptable $\mathrm{FN}$ and misdiagnosis (FN+ FP) rates for diagnosis of DVT. Pathways that provided the best balance of desirable and undesirable effects remained at or below a threshold of $20 \mathrm{FN}$ results per 1000 patients tested $(\leq 2 \%)$ and a threshold of 50 misdiagnosed results per 1000 patients tested (FN+FP $\leq 5 \%$ ).

Summary of evidence. We included a total of 36 studies and 10592 patients to inform test accuracy results (Table 5). Additionally, we included 9 studies and 5126 patients to inform the effect of performing a test on patient important outcomes for patients suspected of having DVT. The references for the included studies are as follows: proximal compression ultrasound ${ }^{84-96}$; wholeleg ultrasound ${ }^{97-103}$; serial ultrasound ${ }^{84,88,104-107} ;$ D-dimer. $^{106-119}$

\section{Low PTP/prevalence ( $\leq 10 \%)$}

Question: In a patient population with a low clinical probability of lower extremity DVT, what is the optimal diagnostic strategy to evaluate for suspected first episode DVT?

\section{Recommendation 5a}

The ASH guideline panel recommends using a strategy starting with D-dimer for excluding DVT in a population with low prevalence/PTP ( $\leq 10 \%$ ), followed by proximal lower

Table 5. Summary of results of studies informing sensitivity and specificity of tests for diagnosis of lower extremity DVT

\begin{tabular}{|c|c|c|c|c|}
\hline Test & No. of participants (studies) & Sensitivity $(95 \% \mathrm{Cl})$ & Specificity $(95 \% \mathrm{Cl})$ & Quality of evidence \\
\hline Proximal compression ultrasound & $2889(12)$ & $0.90(0.87-0.93)$ & $0.99(0.98-0.99)$ & High $^{*}, t, \neq$ \\
\hline Whole-leg ultrasound & $1035(7)$ & $0.93(0.89-0.96)$ & $0.98(0.93-0.99)$ & $\mathrm{High}^{\star},+, \neq$ \\
\hline Serial ultrasound & $2415(6)$ & $0.98(0.96-0.99)$ & $0.998(0.993-0.999)$ & $\mathrm{High}^{\star},+, \neq$ \\
\hline D-dimer & $4409(14)$ & $0.96(0.92-0.98)$ & $0.35(0.28-0.43)$ & Moderate ${ }^{\star}, t, \S$ \\
\hline
\end{tabular}

${ }^{*}$ Certainty in evidence not downgraded for risk of bias, although few studies had a combination of reference standards that were judged to be acceptable by a panel of clinical experts.

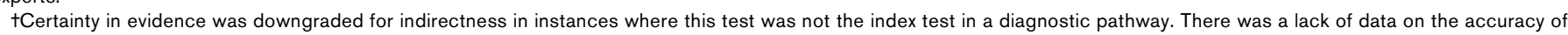

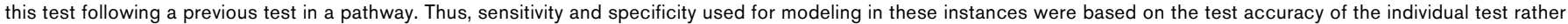
than using the test in a pathway.

¥Minor inconsistency for sensitivity and specificity noted but judged to be insufficient to downgrade the certainty in evidence.

$\S$ Minor inconsistency for sensitivity noted but judged to be insufficient to downgrade the certainty in evidence. Quality of evidence was downgraded for serious unexplained inconsistency in specificity, with range from $16.3 \%$ to $92.2 \%$. Multiple sensitivity analyses could not provide an explanation. 


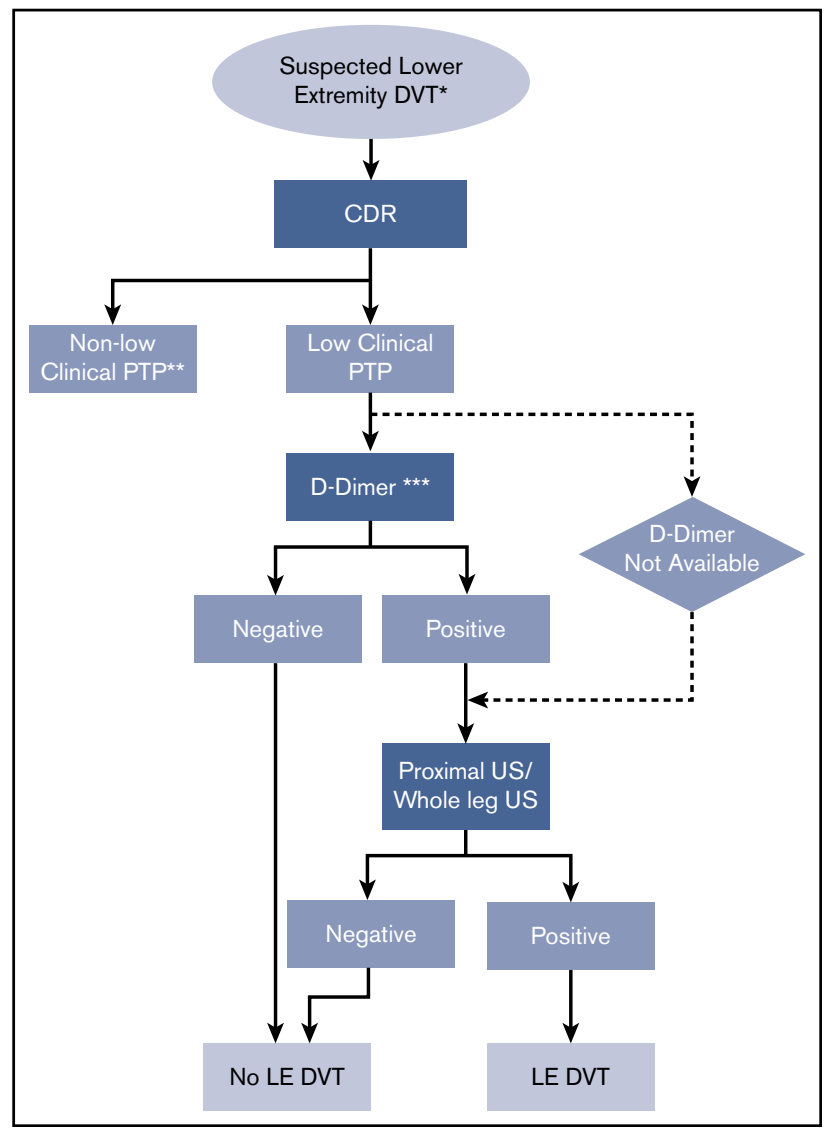

Figure 5. Flowchart for recommendations 5a and b (diagnosis of DVT for patients with low PTP/prevalence [ $\leq \mathbf{1 0 \%}$ ]). *Hemodynamically stable, nonpregnant patient. ${ }^{\star \star}$ See other algorithms. ${ }^{* \star}$ Highly sensitive D-dimer. LE DVT, lower extremity deep vein thrombosis.

extremity ultrasound or whole-leg ultrasound for patients requiring additional testing. If $\mathrm{D}$-dimer is not readily available, alternate acceptable strategies include performing proximal lower extremity or whole-leg ultrasound alone. (Strong recommendation for D-dimer based on moderate certainty in the evidence of effects on clinical outcomes $\oplus \oplus \oplus \bigcirc$ and moderate certainty in the evidence of diagnostic accuracy studies $\oplus \oplus \oplus \bigcirc$; conditional recommendation for proximal or whole-leg ultrasound based on very low certainty in the evidence of effects on clinical outcomes $\oplus 00 \bigcirc$ and moderate certainty in the evidence from diagnostic accuracy studies $\oplus \oplus \oplus \circ)$.

\section{Remarks:}

- Validated clinical decision rules were used to assess clinical probability of DVT in studies evaluating different diagnostic strategies for patients suspected of having a first episode DVT. If a 2-level clinical decision rule is used, this recommendation corresponds to the "unlikely DVT" category.

- A decision to start with D-dimer assumes the results will be obtained in a timely manner and that the cost of D-dimer screening is offset by avoiding unnecessary proximal or whole-leg ultrasound for patients at low PTP for DVT. If the D-dimer strategy is followed, a highly sensitive $\mathrm{D}$-dimer assay is required. A negative D-dimer rules out DVT and no additional testing or anticoagulation is required. D-dimer has limited utility in hospitalized patients and in certain patient populations (postsurgical, pregnant) due to the high frequency of positive Ddimer results with standard thresholds.

- The strategy assumes that test results are obtained under optimal conditions. Suboptimal D-dimer or ultrasound results may require repeat testing.

\section{Recommendation $\mathbf{5 b}$}

The ASH guideline panel recommends against using a positive D-dimer alone to diagnose DVT, and against additional testing following negative proximal or whole-leg ultrasound in a population with low prevalence/PTP $(\leq 10 \%)$. (See grading for recommendation 5a.)

Figure 5 presents a flowchart of recommendations $5 \mathrm{a}$ and $\mathrm{b}$.

Benefits, harms, and burden. For a population with low clinical probability of DVT, proximal or whole-leg ultrasound used as a sole diagnostic tool was adequate for meeting the preestablished threshold criteria. The addition of subsequent tests such as D-dimer or serial ultrasound following initial ultrasound met criteria but added additional testing to the pathway. Pathways with no follow-up testing for positive Ddimer patients resulted in a large number of FP results. Pathways starting with D-dimer where positive results were followed by compression ultrasound, serial ultrasound, or whole-leg ultrasound met threshold criteria. The EtD framework is shown at: https://dbep.gradepro.org/ profile/c039b042-6668-4b66-b2a8-cbd6e0397f9c.

\section{Intermediate PTP/prevalence ( 25\%)}

Question: In a patient population with an intermediate clinical probability of lower extremity DVT, what is the optimal diagnostic strategy to evaluate for suspected first episode DVT?

\section{Recommendation $6 a$}

The ASH guideline panel suggests using a strategy using whole-leg ultrasound, or starting with proximal lower extremity ultrasound for evaluating patients suspected of having DVT in a population with intermediate prevalence/ PTP ( 25\%). No further testing is required if the whole-leg ultrasound is negative, but a negative initial proximal ultrasound should be followed by serial proximal ultrasound if no alternative diagnosis is identified. In an intermediate PTP population where the prevalence is lower, other potentially acceptable strategies include proximal lower extremity ultrasound alone with no additional follow-up testing for negative results, or a strategy starting with $\mathrm{D}$-dimer for excluding DVT followed by proximal lower extremity ultrasound or whole-leg ultrasound for patients requiring additional testing. (Conditional recommendation for proximal or whole-leg ultrasound 


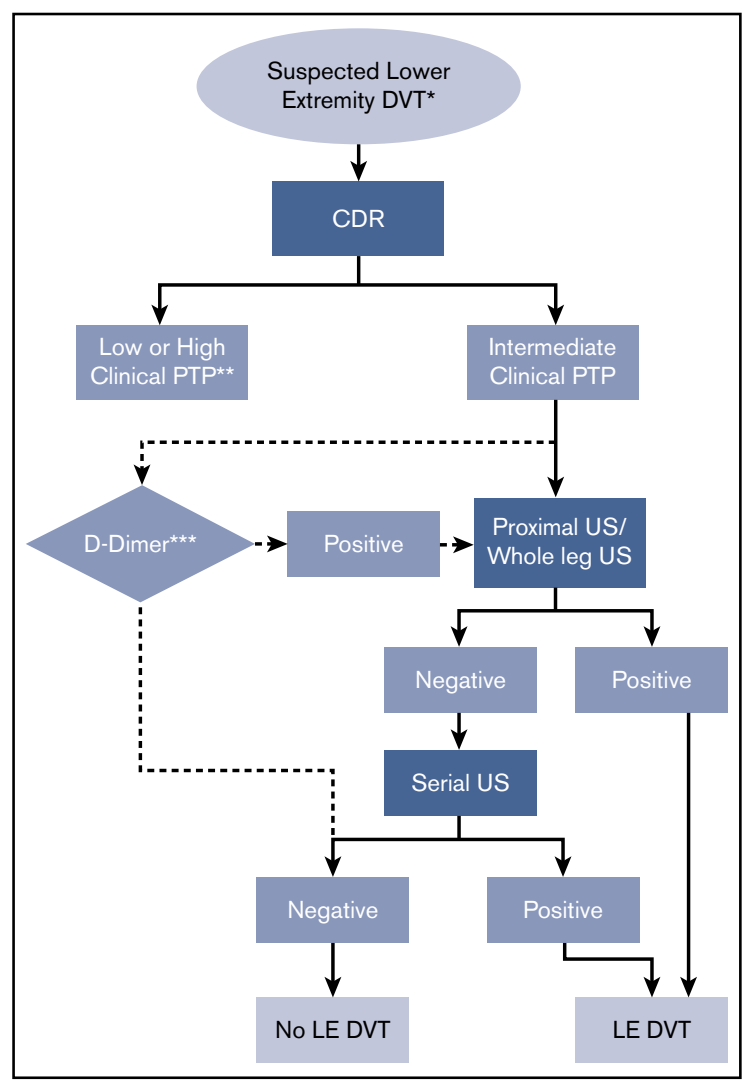

Figure 6. Flowchart for recommendations $6 a$ and $b$ (diagnosis of DVT for patients with intermediate PTP/prevalence $[\sim 25 \%]$ ). *Hemodynamically stable, nonpregnant patient. ${ }^{*}$ See other algorithms. ${ }^{* *}$ Highly sensitive D-dimer.

based on very low certainty in the evidence of effects on clinical outcomes $\oplus 0 \circ \mathrm{O}$ and high certainty in the evidence from diagnostic accuracy studies $\oplus \oplus \oplus \oplus$; conditional recommendation for D-dimer based on moderate certainty in the evidence of effects on clinical outcomes $\oplus \oplus \oplus \bigcirc$ and moderate certainty in the evidence from diagnostic accuracy studies $\oplus \oplus \oplus \mathrm{)})$.

\section{Remarks:}

- Validated clinical decision rules were used to assess clinical probability of DVT in studies evaluating different diagnostic strategies for patients suspected of having a first episode DVT.

- Studies where patients are determined to have an intermediate PTP for DVT have prevalence estimates of $\sim 13 \%$ to $24 \%{ }^{26} \mathrm{~A}$ decision to use a D-dimer strategy in an intermediate PTP population assumes the prevalence is $\sim 15 \%$ or less. A decision to start with D-dimer assumes the results will be obtained in a timely manner and that the cost of D-dimer screening is offset by avoiding unnecessary ultrasound for patients at intermediate PTP for DVT. If the D-dimer strategy is followed, a highly sensitive D-dimer assay is required. A negative D-dimer rules out DVT and no additional testing or anticoagulation is required. D-dimer has limited utility in hospitalized patients and in certain patient populations (postsurgical, pregnant) due to the high frequency of positive Ddimer results with standard thresholds.

- The strategy assumes that test results are obtained under optimal conditions. Suboptimal D-dimer or ultrasound results may require repeat testing.

- Serial ultrasound is defined as 1 additional ultrasound in 1 week following the initial ultrasound.

\section{Recommendation 6b}

The ASH guideline panel recommends against using a positive D-dimer alone to diagnose DVT in a population with intermediate prevalence/PTP $(\sim 25 \%)$. (See grading for recommendation 6a.)

Figure 6 presents a flowchart of recommendations $6 \mathrm{a}$ and $\mathrm{b}$.

Benefits, harms, and burden. For a population with intermediate clinical probability of DVT where the prevalence was modeled at $25 \%$, whole-leg ultrasound as the sole diagnostic tool met the threshold criteria, or proximal lower extremity ultrasound as long as negative results were followed by serial proximal ultrasound. At a lower intermediate prevalence of $15 \%$, proximal ultrasound alone was acceptable, as was use of $\mathrm{D}$-dimer followed by proximal or whole-leg ultrasound. Any pathways with no follow-up testing for positive Ddimer results yielded a large number of FP results. Pathways starting with D-dimer resulted in a large number of FP results despite followup testing for positive D-dimer results.

Intermediate probability encompasses a range of prevalence estimates; at lower DVT prevalences of $15 \%$ or less, threshold criteria for acceptability were met by pathways using D-dimer to exclude a diagnosis of DVT, and pathways using proximal or whole-leg ultrasound as the only diagnostic test. The EtD framework is shown at: https://dbep.gradepro.org/ profile/6e603c3b-fdf2-478b-9abb-bf09ea983839.

\section{High PTP/prevalence ( $\geq 50 \%$ )}

Question: In a patient population with a high clinical probability of lower extremity DVT, what is the optimal diagnostic strategy to evaluate for suspected first episode DVT?

\section{Recommendation 7a}

The ASH guideline panel suggests using a strategy starting with proximal lower extremity or whole-leg ultrasound for assessing patients suspected of having DVT in a population with high prevalence/PTP ( $\geq 50 \%)$. This should be followed by serial ultrasound if the initial ultrasound is negative and no alternative diagnosis is identified. (Conditional recommendation for proximal or whole-leg ultrasound based on very low certainty in the evidence of effects on clinical outcomes $\oplus \mathrm{OOO}$ and high certainty in the evidence from diagnostic accuracy studies $\oplus \oplus \oplus \oplus)$. 


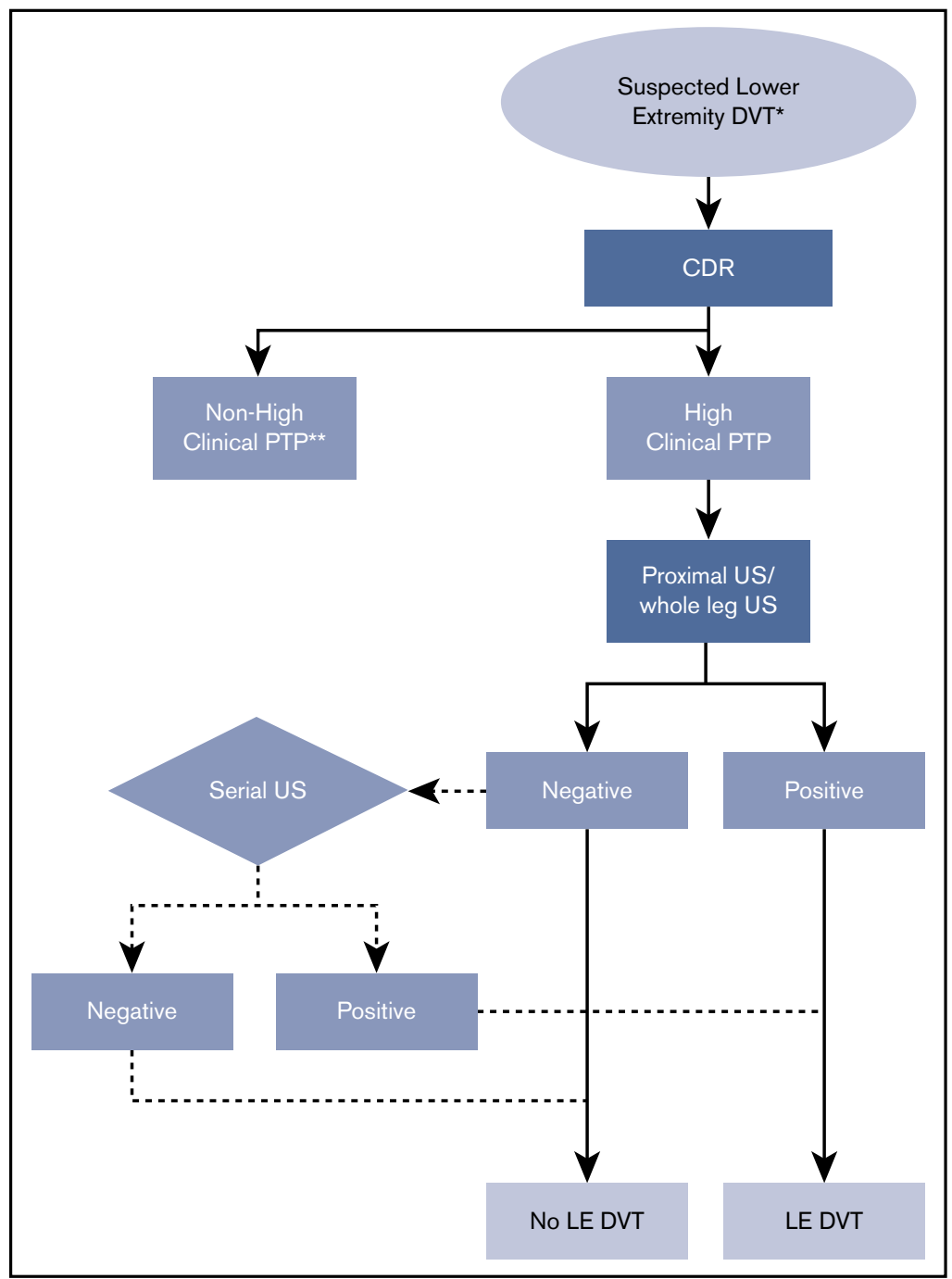

Figure 7. Flowchart for recommendations $7 a$ and $b$ (diagnosis of

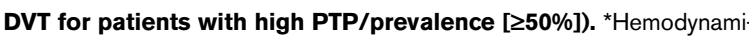
cally stable, nonpregnant patient. ${ }^{*}$ See other algorithms.

\section{Remarks:}

- Validated clinical decision rules were used to assess clinical probability of DVT in studies evaluating different diagnostic strategies for patients suspected of having a first episode DVT. If a 2-level clinical decision rule is used, this recommendation corresponds to the "likely DVT" category.

- The strategy assumes that test results are obtained under optimal conditions. Suboptimal ultrasound results may require repeat testing.

\section{Recommendation 7b}

The ASH guideline panel recommends against using a positive $\mathrm{D}$ dimer alone to diagnose DVT in a population with high prevalence/ PTP $(\geq 50 \%)$. (See grading for recommendation $7 \mathrm{a}$.)

Figure 7 presents a flowchart of recommendations $7 \mathrm{a}$ and $\mathrm{b}$.
Benefits, harms, and burden. For a population with high clinical probability of DVT, proximal or whole-leg ultrasound used as the sole diagnostic tool was not adequate for meeting the threshold criteria. The addition of subsequent tests such as D-dimer or serial ultrasound made the pathway acceptable, as long as positive D-dimer results were followed up with serial ultrasound. Pathways with no follow-up testing for positive D-dimer results yielded a large number of FP results. Pathways starting with D-dimer resulted in a large number of FN results despite follow-up testing for positive D-dimer results. The EtD framework is shown at: https://dbep. gradepro.org/profile/514261d1-957b-4bd0-a137-74cb6878e1f1.

\section{Recurrent DVT (lower extremity)}

Question: For patients with a prior history of DVT, what is the optimal diagnostic strategy to evaluate for suspected recurrent DVT?

\section{Recommendation 8}

The ASH guideline panel suggests using a strategy starting with D-dimer for excluding recurrent DVT in a population with unlikely PTP. Patients with positive D-dimer or those who have 


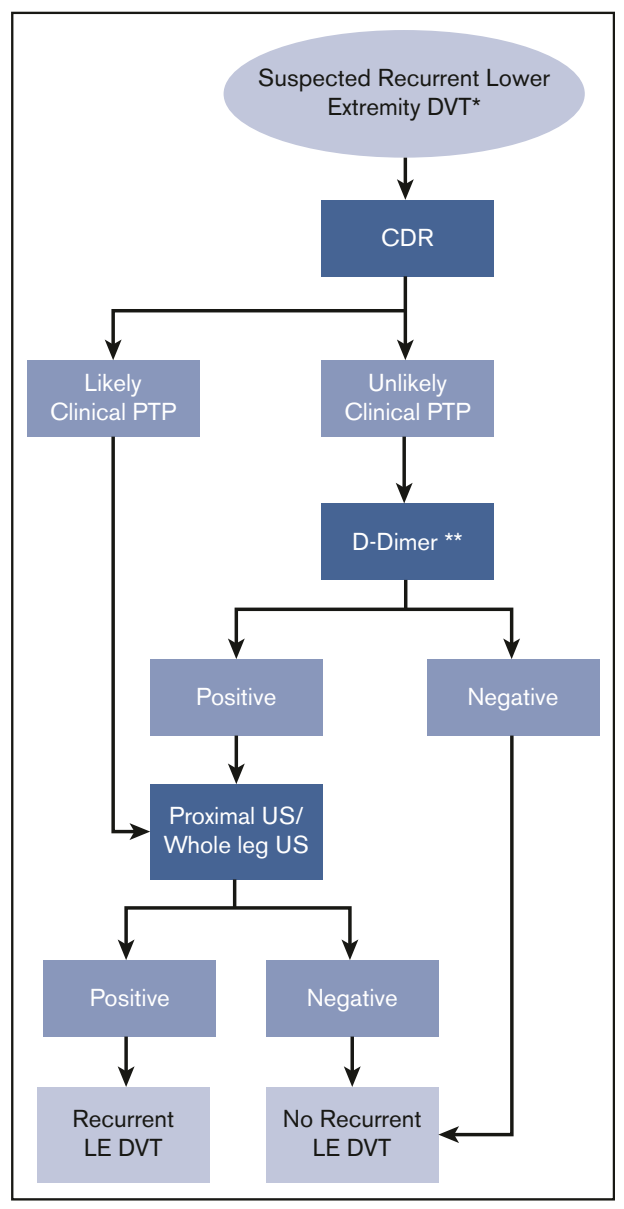

Figure 8. Flowchart for recommendation 8 (diagnosis of recurrent lower

extremity DVT). *Hemodynamically stable, nonpregnant patient. **Highly sensitive D-dimer.

likely PTP should undergo proximal lower extremity ultrasound. (Conditional recommendation for D-dimer and ultrasound based on low certainty in the evidence of effects on clinical outcomes $\oplus \oplus \bigcirc \bigcirc$ and low certainty in the evidence from diagnostic accuracy studies $\oplus \oplus \circ)$ ).

\section{Remarks:}

- In studies assessing this diagnostic strategy for patients suspected of having recurrent DVT, a modified Wells score was used to assess clinical probability of suspected recurrent DVT.

- A decision to start with D-dimer assumes the results will be obtained in a timely manner and that the cost of D-dimer screening is offset by avoiding unnecessary ultrasound for patients at unlikely PTP for DVT. If the D-dimer strategy is followed, a highly sensitive D-dimer assay is required. A negative D-dimer rules out DVT and no additional testing or anticoagulation is required. D-dimer has limited utility in hospitalized patients and in certain patient populations (postsurgical, pregnant) due to the high frequency of positive D-dimer results with standard thresholds. There are limited data on the utility of D-dimer for patients receiving anticoagulant therapy who present with suspected recurrent DVT.

- The strategy assumes that test results are obtained under optimal conditions. Suboptimal D-dimer or ultrasound results may require repeat testing.

- If clinical suspicion remains high following a negative initial ultrasound, serial ultrasound could be considered.

- If prior imaging is available, comparison of the previous and current imaging is warranted to determine whether the findings are new and represent recurrent PE. Studies evaluating compression ultrasound findings of recurrent DVT generally consider involvement of a new venous segment or increase in noncompressibility of $>4 \mathrm{~mm}$ as diagnostic of recurrent DVT.

Figure 8 presents a flowchart of recommendations $8 \mathrm{a}$ and $\mathrm{b}$.

Summary of evidence. We included a total of 2 studies and 310 patients to inform the optimal diagnostic algorithm for patients suspected of recurrent DVT (Table 6).

Benefits, harms, and burden. For a population with suspected recurrent DVT, no modeling of outcomes was performed and recommendations are based on diagnostic pathways used in management studies. The EtD framework is shown at: https://dbep.gradepro.org/profile/37638e9e-85a2499b-8aed-44e2ef55806b.

Other EtD criteria and considerations for DVT (lower extremity) recommendations. None.

Conclusions and research needs for DVT (lower extremity) recommendations. The guideline panel determined that there is high certainty evidence for using D-dimer to exclude DVT for patients with low prevalence/PTP. In an intermediate prevalence population with a prevalence of $\sim 15 \%$, there is moderate certainty evidence for D-dimer, but as the prevalence increases, the utility of D-dimer decreases. There is low to very low certainty evidence for using D-dimer as the initial test for patients with suspected recurrent DVT who are determined to

Table 6. Summary of results of studies informing sensitivity and specificity of tests for diagnosis of recurrent DVT

\begin{tabular}{|c|c|c|c|c|}
\hline Test & No. of participants (studies) & Sensitivity $(95 \% \mathrm{Cl})$ & Specificity $(95 \% \mathrm{Cl})$ & Quality of evidence \\
\hline $\begin{array}{l}\text { D-dimer for low PTP, proximal ultrasound for low } \\
\text { PTP with positive D-dimer and high PTP }\end{array}$ & $105(1)$ & $0.97(0.88-0.99)$ & $0.99(0.86-1.00)$ & Low $^{*}$ \\
\hline Proximal ultrasound & $205(1)$ & $0.91(0.80-0.96)$ & $1.00(0.95-1.00)$ & Lowt \\
\hline Serial ultrasound & $205(1)$ & $0.96(0.86-0.99)$ & $1.00(0.95-1.00)$ & Lowt \\
\hline
\end{tabular}

${ }^{*}$ Certainty in evidence was downgraded for imprecision given the small population size from the 1 recurrent lower extremity DVT study identified for analysis.

tCertainty in evidence was downgraded for imprecision given the small population size from the 1 recurrent lower extremity DVT study identified for analysis. 
Table 7. Summary of results of studies informing sensitivity and specificity of tests for diagnosis of upper extremity DVT

\begin{tabular}{|c|c|c|c|c|}
\hline Test & No. of participants (studies) & Sensitivity $(95 \% \mathrm{Cl})$ & Specificity $(95 \% \mathrm{Cl})$ & Quality of evidence \\
\hline Duplex ultrasound & $465(7)$ & $0.87(0.73-0.94)$ & $0.85(0.72-0.93)$ & Low $^{*}, t, \neq, \S$ \\
\hline D-dimer & 482 (3) & $0.96(0.87-0.99)$ & $0.47(0.43-0.52)$ & Moderate*,,$\neq, \neq, \|$ \\
\hline
\end{tabular}

${ }^{*}$ Certainty in evidence not downgraded for risk of bias, although few studies had a combination of reference standards that were judged to be acceptable by a panel of clinical experts.

tCertainty in evidence was downgraded for indirectness in instances where this test was not the index test in a diagnostic pathway. There was a lack of data on the accuracy of this test

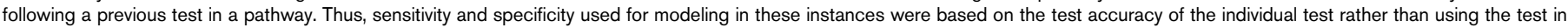
a pathway.

₹Quality of evidence was downgraded for imprecision given small population size.

§Quality of evidence was downgraded for serious unexplained inconsistency in specificity, with range from $55.8 \%$ to $97.5 \%$. Multiple sensitivity analyses could not provide an explanation Quality of evidence was downgraded for serious unexplained inconsistency in specificity, with range from $50.0 \%$ to $97.8 \%$. Multiple sensitivity analyses could not provide an explanation. ||Minor inconsistency for sensitivity and specificity noted but judged to be insufficient to downgrade the certainty in evidence.

have an unlikely PTP using a clinical prediction rule. There is moderate certainty evidence for using proximal or whole-leg ultrasound in an intermediate and high prevalence population, with serial proximal ultrasound or additional testing in high prevalence populations. There is a need to validate clinical prediction rules for patients with suspected recurrent DVT, and to validate ultrasound criteria for recurrent DVT for patients with abnormal ultrasound findings to distinguish acute from chronic DVT. Studies are ongoing of newer modalities such as MRI direct thrombus imaging to assess for acute or chronic thrombus. ${ }^{120}$ The clinical relevance of ultrasound detection of distal (calf) vein thrombosis is uncertain, and research into optimal management is needed.

\section{Diagnosis of upper extremity DVT}

Summary of evidence. We included a total of 9 studies and 752 patients to inform estimates of test accuracy (Table 7). Additionally, we included 3 studies and 500 patients to inform estimates of the effects of performing a test on patient important outcomes for patients suspected of having upper extremity DVT. The references for the included studies are as follows: duplex ultrasound, ${ }^{121-127}$ D-dimer. ${ }^{124,128,129}$

\section{Unlikely PTP/prevalence (10\%)}

Question: In a patient population with an unlikely clinical probability of upper extremity DVT, what is the optimal diagnostic strategy to evaluate for suspected upper extremity DVT?

\section{Recommendation 9a}

The ASH guideline panel suggests a strategy starting with D-dimer for excluding upper extremity DVT in a population with low prevalence/unlikely PTP (10\%), followed by duplex ultrasound if $\mathrm{D}$-dimer is positive. If $\mathrm{D}$-dimer is not readily available, performing duplex ultrasound alone is acceptable. (Conditional recommendation for $\mathrm{D}$-dimer based on very low certainty in the evidence of effects on clinical outcomes $\oplus \bigcirc \bigcirc \bigcirc$ and moderate certainty in the evidence from diagnostic accuracy studies $\oplus \oplus \oplus \bigcirc$; conditional recommendation for duplex ultrasound based on very low certainty in the evidence of effects on clinical outcomes $\oplus \circ \bigcirc \bigcirc$ and low certainty in the evidence from diagnostic accuracy studies $\oplus \oplus \bigcirc)$.

\section{Remarks:}

- In studies assessing this diagnostic strategy for patients suspected of having upper extremity DVT, a dichotomized Constans score (where score $\leq 1$ is unlikely and $\geq 2$ is likely) was used to assess clinical probability of suspected upper extremity DVT.

- A decision to start with D-dimer assumes the results will be obtained in a timely manner and that the cost of D-dimer screening is offset by avoiding unnecessary duplex ultrasound for patients at unlikely PTP for upper extremity DVT. If the $\mathrm{D}$-dimer strategy is followed, a highly sensitive D-dimer assay is required. A negative D-dimer rules out upper extremity DVT and no additional testing or anticoagulation is required. Ddimer has limited utility in hospitalized patients and in certain patient populations (postsurgical, pregnancy) due to the high frequency of positive D-dimer results with standard thresholds.

- The strategy assumes that test results are obtained under optimal conditions. Suboptimal D-dimer or duplex ultrasound results may require repeat testing.

- If clinical suspicion remains high following a negative initial duplex ultrasound, serial duplex ultrasound could be considered.

\section{Recommendation 9b}

The ASH guideline panel recommends against using a positive D-dimer alone to diagnose upper extremity DVT in a population with low prevalence/unlikely PTP (10\%). (See grading for recommendation 9a.)

Figure 9 presents a flowchart of recommendations $9 a$ and $b$.

Benefits, harms, and burden. The panel judged the ranking of pathways based on predetermined thresholds of acceptable FN and misdiagnosis $(\mathrm{FN}+\mathrm{FP})$ rates for diagnosis of upper extremity DVT. Pathways that provided the best balance of desirable and undesirable effects remained at or below a threshold of $50 \mathrm{FN}$ results per 1000 patients tested $(\leq 5 \%)$ and a threshold of 100 misdiagnosed results per 1000 patients tested (FN+FP $\leq 10 \%$ ).

For a population with low clinical probability of upper extremity DVT, acceptability criteria were met by pathways beginning with D-dimer where D-dimer positive results were followed by a single duplex 
Figure 9. Flowchart for recommendations $9 a$ and $b$ (diagnosis of upper extremity DVT for patients with unlikely PTP/low prevalence [10\%]). *Hemodynamically stable, nonpregnant patient. ${ }^{* \star}$ See other algorithms. ${ }^{* \star \star}$ Highly sensitive D-dimer. UE DVT, upper extremity deep vein thrombosis.

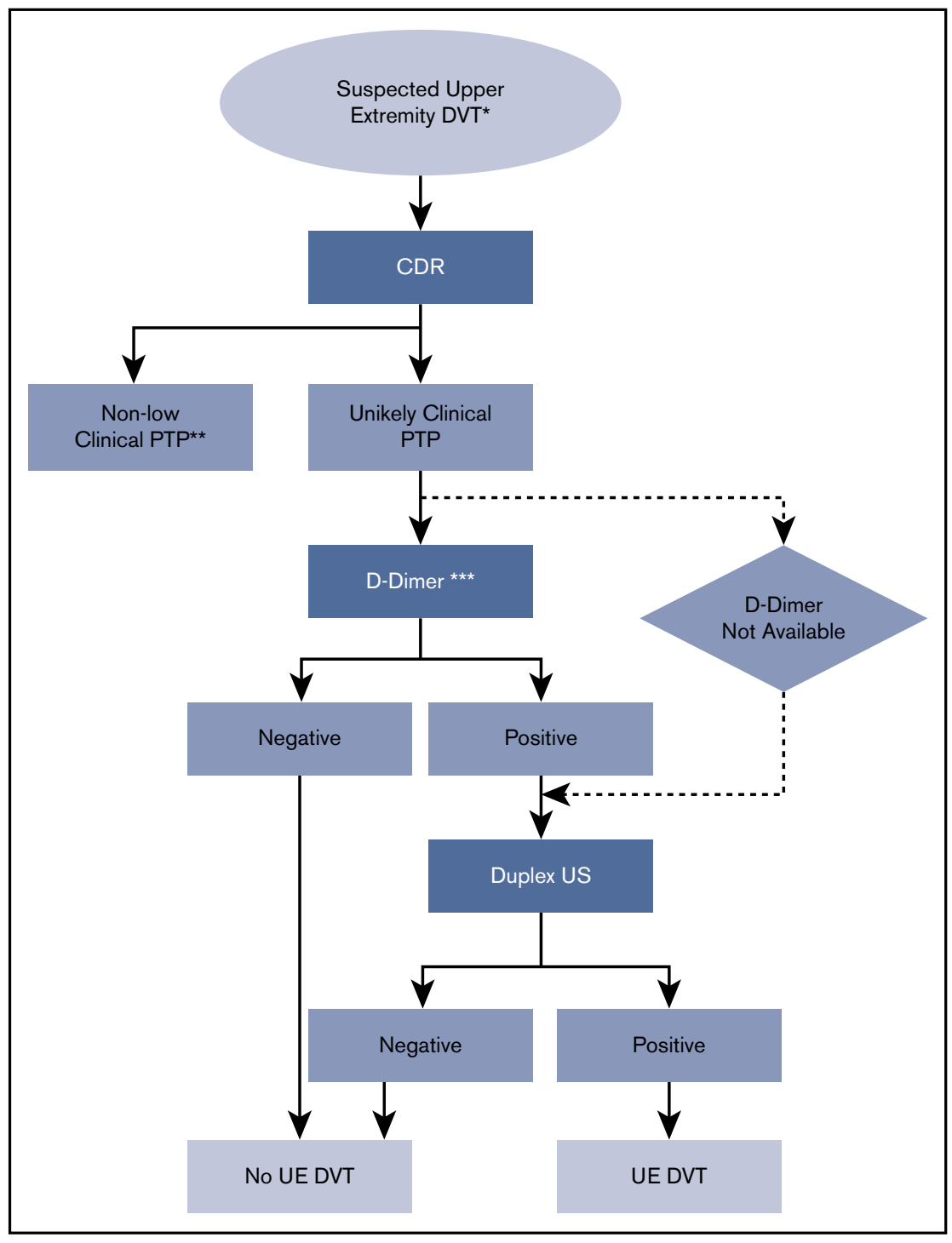

ultrasound. Duplex ultrasound used as the sole diagnostic tool was not adequate for meeting the threshold criteria for acceptability but was felt by the panel to be clinically acceptable. The addition of subsequent tests following duplex ultrasound including D-dimer or serial ultrasound similarly did not meet acceptability criteria. Pathways with no follow-up testing for positive D-dimer patients resulted in a large number of positive results. The EtD framework is shown at: https://dbep.gradepro. org/profile/2faee274-8c28-4f19-b8a2-23947d6f29fc.

\section{Likely PTP/prevalence (40\%)}

Question: In a patient population with a likely clinical probability of upper extremity DVT, what is the optimal diagnostic strategy to evaluate for suspected upper extremity DVT?

\section{Recommendation 10a}

The ASH guideline panel suggests a strategy of either D-dimer followed by duplex ultrasound/serial duplex ultrasound, or duplex ultrasound/serial duplex ultrasound alone for assessing patients suspected of having upper extremity DVT in a population with high prevalence/likely PTP (40\%). (Conditional recommendation for $\mathrm{D}$-dimer based on very low certainty in the evidence of effects on clinical outcomes $\oplus \bigcirc \bigcirc \bigcirc$ and moderate certainty in the evidence from diagnostic accuracy studies $\oplus \oplus \oplus \bigcirc$; conditional recommendation for duplex ultrasound based on very low certainty in the evidence of effects on clinical outcomes $\oplus 00 \bigcirc$ and low certainty in the evidence from diagnostic accuracy studies $\oplus \oplus \bigcirc)$.

\section{Remarks:}

- In studies assessing this diagnostic strategy for patients suspected of having upper extremity DVT, a dichotomized Constans score (where score $\leq 1$ is unlikely and $\geq 2$ is likely) was used to assess clinical probability of suspected upper extremity DVT. 


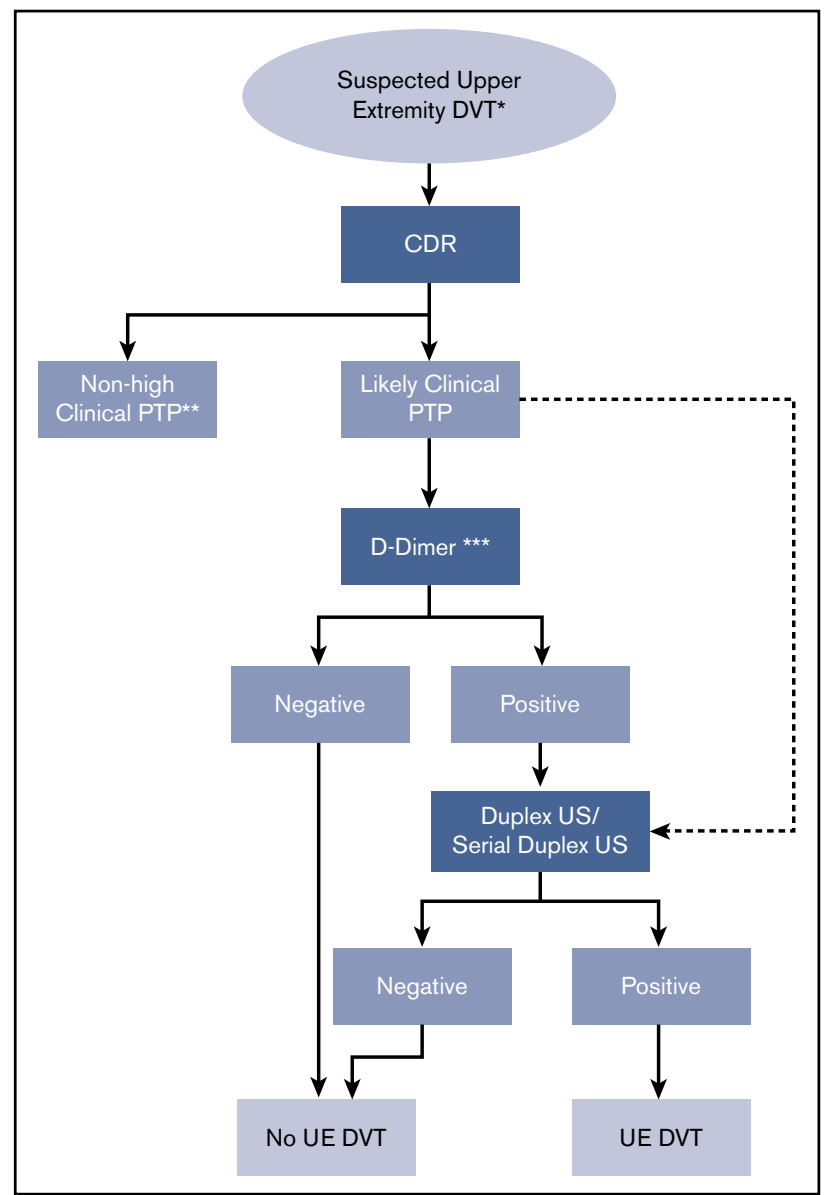

Figure 10. Flowchart for recommendations 10a and $b$ (diagnosis of upper extremity DVT for patients with likely PTP/high prevalence [40\%]). *Hemodynamically stable, nonpregnant patient. ${ }^{*}$ See other algorithms. ${ }^{* \star *}$ Highly sensitive D-dimer.

- A decision to start with D-dimer assumes the results will be obtained in a timely manner and that the cost of D-dimer screening is offset by avoiding unnecessary duplex US. If the D-dimer strategy is followed, a highly sensitive D-dimer assay is required. A negative D-dimer rules out upper extremity DVT and no additional testing or anticoagulation is required. D-dimer has limited utility in hospitalized patients and in certain patient populations (postsurgical, pregnant) due to the high frequency of positive D-dimer results with standard thresholds.

- The strategy assumes that test results are obtained under optimal conditions. Suboptimal D-dimer or duplex ultrasound results may require repeat testing.

\section{Recommendation 10b}

The ASH guideline panel recommends against using a positive D-dimer alone to diagnose upper extremity DVT in a population with high prevalence/likely PTP (40\%). (See grading for recommendation 10a.)
Figure 10 presents a flowchart of recommendations $10 \mathrm{a}$ and $\mathrm{b}$.

Benefits, harms, and burden. The panel judged the ranking of pathways based on predetermined threshold of acceptable FN and misdiagnosis (FN+FP) rates for diagnosis of upper extremity DVT. Pathways that provided the best balance of desirable and undesirable effects remained at or below a threshold of $50 \mathrm{FN}$ results per 1000 patients tested $(\leq 5 \%)$ and a threshold of 100 misdiagnosed results per 1000 patients tested ( $F N+F P \leq 10 \%)$. If no pathways met these criteria, a threshold of $100 \mathrm{FN}$ results per 1000 patients tested $(\leq 10 \%)$ and a new threshold of 200 misdiagnosed results per 1000 patients tested (FN+FP $\leq 20 \%$ ) was used.

For a population with high clinical probability of upper extremity DVT, none of the evaluated pathways met the initial threshold criteria set by the panel. Pathways were then reviewed for meeting the new parameters, as described in the previous paragraph. Duplex ultrasound used as the sole diagnostic tool was not adequate for meeting the threshold criteria. The addition of subsequent tests including D-dimer or serial ultrasound were acceptable, as long as positive D-dimer results were followed up with serial ultrasound. Ultrasound similarly did not meet acceptability criteria. Any pathways with no follow-up testing for positive D-dimer results yielded a large number of FP results. The EtD framework is shown at: https://dbep.gradepro.org/profile/ c2321da4-0035-4e74-b6da-f49bbc12b1de.

Other EtD criteria and considerations for upper extremity DVT recommendations. None.

Conclusions and research needs for upper extremity DVT recommendations. The guideline panel determined that there is low certainty evidence for using D-dimer to exclude upper extremity DVT for patients with low PTP/prevalence. There is moderate certainty evidence for using duplex ultrasound for patients with a positive D-dimer and for patients with high prevalence. There is a need to further evaluate clinical prediction rules when assessing patients with suspected upper extremity DVT.

\section{What are others saying and what is new in these ASH guidelines?}

The American College of Chest Physicians (ACCP) EvidenceBased Clinical Practice Guidelines chapter on diagnosis of DVT suggests that clinical assessment of PTP of DVT, rather than performing the same tests in all patients, should guide the diagnostic process for a first lower extremity DVT (Grade 2B). ${ }^{130}$ The ACCP recommendations are as follows (p. e351S):

In patients with a low pretest probability of first lower extremity DVT, we recommend initial testing with D-dimer or ultrasound (US) of the proximal veins over no diagnostic testing (Grade 1B), venography (Grade 1B), or whole-leg US (Grade 2B). In patients with moderate pretest probability, we recommend initial testing with a highly sensitive D-dimer, proximal compression US, or whole-leg US rather than no testing (Grade 1B) or venography (Grade 1B). In patients with a high pretest probability, we recommend proximal compression or whole-leg US over no testing (Grade 1B) or venography (Grade 1B).

In the high PTP population, the ACCP recommendations are as follows (p. e353S): 
In patients with a negative proximal CUS, we recommend additional testing with a highly sensitive D-dimer or wholeleg US or repeat proximal CUS in 1 week over no further testing (Grade 1B for all comparisons) or venography (Grade 2B for all comparisons). We recommend that patients with a single negative proximal CUS and positive D-dimer undergo whole-leg US or repeat proximal CUS in 1 week over no further testing (Grade 1B) or venography (Grade $2 B$ ). In patients with negative serial proximal CUS, a negative single proximal CUS and negative highly sensitive D-dimer, or a negative whole-leg US, we recommend no further testing over venography or additional US (Grade 1B for negative serial proximal CUS and for negative single proximal CUS and highly sensitive D-dimer; Grade 2B for negative whole-leg US). ${ }^{130}$

There are no ACCP recommendations on diagnosis of $\mathrm{PE}$.

Both the ACCP and these guidelines are based on systematic reviews of the literature. In contrast to the ACCP guidelines, we used diagnostic test accuracy estimates and population prevalences to model the various diagnostic pathways to determine the optimal diagnostic pathway and compared the model estimates, where available, to published results. Variations in the recommendations are attributable to the population prevalence used in the model. The model was sensitive to these changes and yielded similar results when compared with management studies evaluating the same diagnostic pathway.

\section{Limitations of these guidelines}

These guidelines are based on modeled estimates for the population prevalence of VTE. Prevalence and PTP are continuous variables but for modeling purposes, required input of a specific population prevalence. For patients at intermediate risk of PE or DVT, this resulted in a range of potential prevalences, which could affect the recommendation. The availability of diagnostic tests and the expertise to interpret the results is variable and may affect how these guidelines will be used.

\section{Revision or adaptation of the guidelines}

\section{Plans for updating these guidelines}

After publication of these guidelines, ASH will maintain them through surveillance for new evidence, ongoing review by experts, and regular revisions.

\section{Updating or adapting recommendations locally}

Adaptation of these guidelines will be necessary in many circumstances. These adaptations should be based on the associated EtD frameworks. ${ }^{131}$

\section{Acknowledgments}

The authors acknowledge John Bartholomew for participation on the panel during the initial stages of the guideline development process, and for review of the draft recommendations. The authors acknowledge Carolina Martins, Rasha Khatib, Itziar Etxeandia, Yuan Zhang, David Wooldridge, Ahmad Mustafa, Anchal Sethi, Kelly Orozco, Hani Alturkmani, Mariam Baig, Merrill Thomas, Rakesh Ponnapureddy, Rohan Kehar, Waled Bahaj, Jamie Varghese, and Samantha Eiffert for supporting the systematic reviews; Robin Arnold for other assistance; and Wojtek Wiercioch, Robby Nieuwlaat, and Holger Schünemann for coordination of the systematic review team. H.J.S. prepared a template of these guidelines for all panels that was critically reviewed by Adam Cuker, Rob Kunkle, the ASH Guideline Oversight Subcommittee, the Methods Group supporting the guidelines, and Blood Advances editors.

\section{Authorship}

Contribution: R.A.M. and W.L. wrote the first draft of the manuscript and revised the manuscript based on the authors' suggestions; guideline panel members (G.L.G., S.M.B., M.R., L.B.H., E.L., J.A.K., S.C., M.S., and H.J.S.) critically reviewed the manuscript and provided suggestions for improvement; members of the knowledge synthesis team (Payal Patel, M.B., Parth Patel, C.B., H.B., and W.W.) contributed evidence summaries to the guidelines; W.L. and R.A.M. were the chair and vice chair of the panel and led the panel meeting; and all authors approved of the content.

Conflict-of-interest disclosure: All authors were members of the guideline panel or members of the systematic review team or both. As such, they completed a disclosure of interest form, which was reviewed by $\mathrm{ASH}$ and is available as supplements 2 and 3.

ORCID profiles: W.L., 0000-0003-2508-1786; G.L.G., 00000002-9253-248X; P.P., 0000-0002-4864-4927; W.W., 00000001-6576-1650; R.A.M., 0000-0002-2091-0875.

Correspondence: Wendy Lim, McMaster University, 50 Charlton Ave East, Hamilton, ON L8N 4A6, Canada; e-mail: limwp@ mcmaster.ca.

\section{References}

1. Schünemann HJ, Wiercioch W, Etxeandia I, et al. Guidelines 2.0: systematic development of a comprehensive checklist for a successful guideline enterprise. CMAJ. 2014;186(3):E123-E142.

2. Institute of Medicine (US) Committee on Standards for Developing Trustworthy Clinical Practice Guidelines. Clinical Practice Guidelines We Can Trust. Washington, DC: National Academies Press; 2011.

3. Qaseem A, Forland F, Macbeth F, Ollenschläger G, Phillips S, van der Wees P; Board of Trustees of the Guidelines International Network. Guidelines International Network: toward international standards for clinical practice guidelines. Ann Intern Med. 2012;156(7):525-531.

4. Schünemann HJ, Al-Ansary LA, Forland F, et al; Board of Trustees of the Guidelines International Network. Guidelines International Network: principles for disclosure of interests and management of conflicts in guidelines. Ann Intern Med. 2015;163(7):548-553.

5. Langer G, Meerpohl JJ, Perleth M, Gartlehner G, Kaminski-Hartenthaler A, Schünemann H. GRADE guidelines: 1. Introduction - GRADE evidence profiles and summary of findings tables [in German]. Z Evid Fortbild Qual Gesundhwes. 2012;106(5):357-368. 
6. Guyatt GH, Oxman AD, Vist GE, et al; GRADE Working Group. GRADE: an emerging consensus on rating quality of evidence and strength of recommendations. BMJ. 2008;336(7650):924-926.

7. Rodger MA, Le Gal G, Wells P, et al. Clinical decision rules and D-Dimer in venous thromboembolism: current controversies and future research priorities. Thromb Res. 2014;134(4):763-768.

8. Kline KA, Courtney DM, Kabrhel C, et al. Prospective multicenter evaluation of the pulmonary embolism rule-out criteria. J Thromb Haemost. 2008;6(5): 772-780.

9. van Beek EJ, Brouwerst EM, Song B, Stein PD, Oudkerk M. Clinical validity of a normal pulmonary angiogram in patients with suspected pulmonary embolism--a critical review. Clin Radiol. 2001;56(10):838-842.

10. Hull R, Hirsh J, Sackett DL, et al. Clinical validity of a negative venogram in patients with clinically suspected venous thrombosis. Circulation. 1981;64(3): 622-625.

11. Lo B, Fields M. Conflict of Interest in Medical Research, Education, and Practice. Washington, DC: National Academic Press, Institute of Medicine; 2009.

12. Akl EA, El-Hachem P, Abou-Haidar H, Neumann I, Schünemann HJ, Guyatt GH. Considering intellectual, in addition to financial, conflicts of interest proved important in a clinical practice guideline: a descriptive study. J Clin Epidemiol. 2014;67(11):1222-1228.

13. Guyatt G, AkI EA, Hirsh J, et al. The vexing problem of guidelines and conflict of interest: a potential solution. Ann Intern Med. 2010;152(11):738-741.

14. Schünemann HJ, Osborne M, Moss J, et al; ATS Ethics and Conflict of Interest Committee and the Documents Development and Implementation Committee. An official American Thoracic Society Policy statement: managing conflict of interest in professional societies. Am J Respir Crit Care Med. 2009;180(6):564-580.

15. Brożek J, Nowak A, Kunstman P, Schünemann HJ. GRADEpro Guideline Development Tool (G2DT). Available at: www.guidelinedevelopment.org. Accessed 18 May 2018.

16. Douma RA, Mos IC, Erkens PM, et al; Prometheus Study Group. Performance of 4 clinical decision rules in the diagnostic management of acute pulmonary embolism: a prospective cohort study. Ann Intern Med. 2011;154(11):709-718.

17. Guyatt $\mathrm{GH}$, Oxman AD, Kunz R, et al. GRADE guidelines: 2. Framing the question and deciding on important outcomes. J Clin Epidemiol. 2011;64(4): 395-400.

18. Guyatt G, Oxman AD, Akl EA, et al. GRADE guidelines: 1. Introduction-GRADE evidence profiles and summary of findings tables. J Clin Epidemiol. 2011; 64(4):383-394.

19. Schünemann HJ, Mustafa R, Brozek J, et al; GRADE Working Group. GRADE Guidelines: 16. GRADE evidence to decision frameworks for tests in clinical practice and public health. J Clin Epidemiol. 2016;76:89-98.

20. Alonso-Coello P, Oxman AD, Moberg J, et al; GRADE Working Group. GRADE Evidence to Decision (EtD) frameworks: a systematic and transparent approach to making well informed healthcare choices. 2: Clinical practice guidelines. BMJ. 2016;353:i2089.

21. Alonso-Coello P, Schünemann HJ, Moberg J, et al; GRADE Working Group. GRADE Evidence to Decision (EtD) frameworks: a systematic and transparent approach to making well informed healthcare choices. 1: Introduction. BMJ. 2016;353:i2016.

22. Whiting PF, Rutjes AW, Westwood ME, et al; QUADAS-2 Group. QUADAS-2: a revised tool for the quality assessment of diagnostic accuracy studies. Ann Intern Med. 2011;155(8):529-536.

23. Atkins D, Eccles M, Flottorp S, et al; GRADE Working Group. Systems for grading the quality of evidence and the strength of recommendations I: critical appraisal of existing approaches The GRADE Working Group. BMC Health Serv Res. 2004;4(1):38.

24. Schünemann HJ, Oxman AD, Brozek J, et al; GRADE Working Group. Grading quality of evidence and strength of recommendations for diagnostic tests and strategies [published correction appears in BMJ. 2008;336(7654)]. BMJ. 2008;336(7653):1106-1110.

25. Ceriani E, Combescure C, Le Gal G, et al. Clinical prediction rules for pulmonary embolism: a systematic review and meta-analysis. $J$ Thromb Haemost. 2010;8(5):957-970.

26. Geersing GJ, Zuithoff NP, Kearon C, et al. Exclusion of deep vein thrombosis using the Wells rule in clinically important subgroups: individual patient data meta-analysis. BMJ. 2014;348:g1340.

27. Carrier M, Rodger MA, Wells PS, Righini M, Le Gal G. Residual vein obstruction to predict the risk of recurrent venous thromboembolism in patients with deep vein thrombosis: a systematic review and meta-analysis. J Thromb Haemost. 2011;9(6):1119-1125.

28. Anderson DR, Kahn SR, Rodger MA, et al. Computed tomographic pulmonary angiography vs ventilation-perfusion lung scanning in patients with suspected pulmonary embolism: a randomized controlled trial. JAMA. 2007;298(23):2743-2753.

29. Bajc M, Albrechtsson U, Olsson CG, Olsson B, Jonson B. Comparison of ventilation/perfusion scintigraphy and helical CT for diagnosis of pulmonary embolism; strategy using clinical data and ancillary findings. Clin Physiol Funct Imaging. 2002;22(6):392-397.

30. Elias A, Colombier D, Victor G, et al. Diagnostic performance of complete lower limb venous ultrasound in patients with clinically suspected acute pulmonary embolism [published correction appears in Thromb Haemost. 2004;91(3):635]. Thromb Haemost. 2004;91(1):187-195.

31. Eng CW, Wansaicheong G, Goh SK, Earnest A, Sum C. Exclusion of acute pulmonary embolism: computed tomography pulmonary angiogram or D-dimer? Singapore Med J. 2009;50(4):403-406.

32. Hantous-Zannad S, Esseghaier S, Ridène I, et al. Acute pulmonary embolism: epidemiologic and tomodensitometric study. Tunis Med. 2010;88(12): 880-884.

33. Jiménez D, Gómez M, Herrero R, et al. Thromboembolic events in patients after a negative computed tomography pulmonary angiogram: a retrospective study of 165 patients [in Spanish]. Arch Bronconeumol. 2006;42(7):344-348. 
34. Macdonald WB, Patrikeos AP, Thompson RI, Adler BD, van der Schaaf AA. Diagnosis of pulmonary embolism: ventilation perfusion scintigraphy versus helical computed tomography pulmonary angiography. Australas Radiol. 2005;49(1):32-38.

35. Megyeri B, Christe A, Schindera ST, et al. Accuracy of computed tomography angiography in the detection of pulmonary embolism in patients with high body weight. Eur J Intern Med. 2014;25(8):724-730.

36. Pacouret G, Marie O, Alison D, et al. Association of D-dimer and helicoidal thoracic scanner for diagnosis of pulmonary embolism. Prospective study of 106 ambulatory patients [in French]. Presse Med. 2002;31(1 pt 1):13-18.

37. Pérez de Llano LA, Veres Racamonde A, Ortiz Piquer M, et al. Safety of withholding anticoagulant therapy in patients who have clinically suspected pulmonary embolism and negative results on helical computed tomography. Respiration. 2006;73(4):514-519.

38. Perrier A, Roy PM, Sanchez O, et al. Multidetector-row computed tomography in suspected pulmonary embolism. N Engl J Med. 2005;352(17): 1760-1768.

39. Righini M, Le Gal G, Aujesky D, et al. Diagnosis of pulmonary embolism by multidetector CT alone or combined with venous ultrasonography of the leg: a randomised non-inferiority trial. Lancet. 2008;371(9621):1343-1352.

40. Righini M, Van Es J, Den Exter PL, et al. Age-adjusted D-dimer cutoff levels to rule out pulmonary embolism: the ADJUST-PE study. JAMA. 2014;311 (11): 1117-1124.

41. Stein PD, Fowler SE, Goodman LR, et al; PIOPED II Investigators. Multidetector computed tomography for acute pulmonary embolism. N Engl J Med. 2006;354(22):2317-2327.

42. Subramaniam RM, Blair D, Gilbert K, Coltman G, Sleigh J, Karalus N. Withholding anticoagulation after a negative computed tomography pulmonary angiogram as a stand-alone imaging investigation: a prospective management study. Intern Med J. 2007;37(9):624-630.

43. Abcarian PW, Sweet JD, Watabe JT, Yoon HC. Role of a quantitative D-dimer assay in determining the need for CT angiography of acute pulmonary embolism. AJR Am J Roentgenol. 2004;182(6):1377-1381.

44. Bosson JL, Barro C, Satger B, Carpentier PH, Polack B, Pernod G. Quantitative high D-dimer value is predictive of pulmonary embolism occurrence independently of clinical score in a well-defined low risk factor population. J Thromb Haemost. 2005;3(1):93-99.

45. de Moerloose $\mathrm{P}$, Desmarais S, Bounameaux H, et al. Contribution of a new, rapid, individual and quantitative automated D-dimer ELISA to exclude pulmonary embolism. Thromb Haemost. 1996;75(1):11-13.

46. de Monyé W, Sanson BJ, Büller HR, Pattynama PM, Huisman MV; ANTELOPE Study Group. The performance of two rapid quantitative D-dimer assays in 287 patients with clinically suspected pulmonary embolism. Thromb Res. 2002;107(6):283-286.

47. den Exter PL, van Es J, Erkens PM, et al. Impact of delay in clinical presentation on the diagnostic management and prognosis of patients with suspected pulmonary embolism. Am J Respir Crit Care Med. 2013;187(12):1369-1373.

48. Di Nisio M, Sohne M, Kamphuisen PW, Büller HR. D-dimer test in cancer patients with suspected acute pulmonary embolism. $J$ Thromb Haemost. 2005; 3(6):1239-1242.

49. Dunn KL, Wolf JP, Dorfman DM, Fitzpatrick P, Baker JL, Goldhaber SZ. Normal D-dimer levels in emergency department patients suspected of acute pulmonary embolism. J Am Coll Cardiol. 2002;40(8):1475-1478.

50. Emet M, Ozucelik DN, Sahin M, Oran M, Sivri B. Computed tomography pulmonary angiography in the diagnosis of acute pulmonary embolism in the emergency department. Adv Ther. 2007;24(6):1173-1180.

51. Friera-Reyes A, Caballero P, Ruiz-Giménez N, et al; Grupo de Estudio de Enfermedad Tromboembólica Venosa. Usefulness of fast ELISA determination of D-dimer levels for diagnosing pulmonary embolism in an emergency room [in Spanish]. Arch Bronconeumol. 2005;41(9):499-504.

52. Froehling DA, Daniels PR, Swensen SJ, et al. Evaluation of a quantitative D-dimer latex immunoassay for acute pulmonary embolism diagnosed by computed tomographic angiography. Mayo Clin Proc. 2007;82(5):556-560.

53. Ghanima W, Abdelnoor M, Mowinckel MC, Sandset PM. The performance of STA-Liatest D-dimer assay in out-patients with suspected pulmonary embolism. Br J Haematol. 2006;132(2):210-215.

54. Goekoop RJ, Steeghs N, Niessen RW, et al. Simple and safe exclusion of pulmonary embolism in outpatients using quantitative D-dimer and Wells' simplified decision rule. Thromb Haemost. 2007;97(1):146-150.

55. Leclercq MG, Lutisan JG, van Marwijk Kooy M, et al. Ruling out clinically suspected pulmonary embolism by assessment of clinical probability and D-dimer levels: a management study. Thromb Haemost. 2003;89(1):97-103.

56. Oger E, Leroyer C, Bressollette L, et al. Evaluation of a new, rapid, and quantitative D-dimer test in patients with suspected pulmonary embolism. Am J Respir Crit Care Med. 1998;158(1):65-70.

57. Palud L, Laurent $M$, Guéret $P$, et al. Value of the association of $D$-dimer measurement and the evaluation of clinical probability in a non-invasive diagnostic strategy of pulmonary embolism [in French]. Arch Mal Coeur Vaiss. 2004;97(2):93-99.

58. Parent F, Maître S, Meyer G, et al. Diagnostic value of D-dimer in patients with suspected pulmonary embolism: results from a multicentre outcome study. Thromb Res. 2007;120(2):195-200.

59. Perrier A, Desmarais S, Miron MJ, et al. Non-invasive diagnosis of venous thromboembolism in outpatients. Lancet. 1999;353(9148): 190-195.

60. Perrier A, Roy PM, Aujesky D, et al. Diagnosing pulmonary embolism in outpatients with clinical assessment, D-dimer measurement, venous ultrasound, and helical computed tomography: a multicenter management study. Am J Med. 2004;116(5):291-299.

61. Quinn DA, Fogel RB, Smith CD, et al. D-dimers in the diagnosis of pulmonary embolism. Am J Respir Crit Care Med. 1999;159(5):1445-1449. 
62. Sijens PE, van Ingen HE, van Beek EJ, Berghout A, Oudkerk M. Rapid ELISA assay for plasma D-dimer in the diagnosis of segmental and subsegmental pulmonary embolism. A comparison with pulmonary angiography. Thromb Haemost. 2000;84(2):156-159.

63. Söderberg M, Brohult J, Jorfeldt L, Lärfars G. The use of D-dimer testing and Wells score in patients with high probability for acute pulmonary embolism. J Eval Clin Pract. 2009;15(1):129-133.

64. Szturmowicz M, Kacprzak A, Wyrostkiewicz D, et al. Non-high risk PE in the patients with acute or exacerbated respiratory disease: the value of the algorithm based on D-dimer evaluation and Revised Geneva Score. Pneumonol Alergol Pol. 2015;83(6):445-452.

65. Toulon P, Lecourvoisier C. D-dimer testing for suspected pulmonary embolism: performance of two rapid quantitative assays. Lab Med. 2004;35(2): 117-120.

66. Fr V, Hainaut P, Fr T, et al. ELISA D-dimer measurement for the clinical suspicion of pulmonary embolism in the emergency department: one-year observational study of the safety profile and physician's prescription. Acta Clin Belg. 2003;58(4):233-240.

67. Bolado AG, Barcena MV, del Cura JL, Gorrino O, Grande D. Diagnostic indication for venous echo-Doppler of the lower limbs in the diagnosis of thromboembolic disease with suspected pulmonary thromboembolism [in Spanish]. Radiologia (Madr). 2003;45(5):213-218.

68. Guilabert JP, Manzur DN, Tarrasa MJ, Llorens ML, Braun P, Arques MP. Can multislice CT alone rule out reliably pulmonary embolism? A prospective study. Eur J Radiol. 2007;62(2):220-226.

69. Kim KIM, Müller NL, Mayo JR. Clinically suspected pulmonary embolism: utility of spiral CT. Radiology. 1999;210(3):693-697.

70. Mac Gillavry MR, Sanson BJ, Büller HR, Brandjes DP; ANTELOPE-Study Group. Compression ultrasonography of the leg veins in patients with clinically suspected pulmonary embolism: is a more extensive assessment of compressibility useful? Thromb Haemost. 2000;84(6):973-976.

71. Perrier A, Bounameaux H, Morabia A, et al. Diagnosis of pulmonary embolism by a decision analysis-based strategy including clinical probability, D-dimer levels, and ultrasonography: a management study. Arch Intern Med. 1996;156(5):531-536.

72. Turkstra F, Kuijer PM, van Beek EJ, Brandjes DP, ten Cate JW, Büller HR. Diagnostic utility of ultrasonography of leg veins in patients suspected of having pulmonary embolism. Ann Intern Med. 1997;126(10):775-781.

73. Blachere $\mathrm{H}$, Latrabe $\mathrm{V}$, Montaudon $\mathrm{M}$, et al. Pulmonary embolism revealed on helical $\mathrm{CT}$ angiography: comparison with ventilation-perfusion radionuclide lung scanning. AJR Am J Roentgenol. 2000;174(4):1041-1047.

74. Bounameaux $\mathrm{H}$, Cirafici $\mathrm{P}$, de Moerloose $\mathrm{P}$, et al. Measurement of D-dimer in plasma as diagnostic aid in suspected pulmonary embolism. Lancet. 1991; 337(8735):196-200.

75. Freitas JE, Sarosi MG, Nagle CC, Yeomans ME, Freitas AE, Juni JE. Modified PIOPED criteria used in clinical practice. J Nucl Med. 1995;36(9): 1573-1578.

76. Miron MJ, Perrier A, Bounameaux $\mathrm{H}$, et al. Contribution of noninvasive evaluation to the diagnosis of pulmonary embolism in hospitalized patients. Eur Respir J. 1999;13(6):1365-1370.

77. van Rossum AB, Pattynama PM, Mallens WM, Hermans J, Heijerman HG. Can helical CT replace scintigraphy in the diagnostic process in suspected pulmonary embolism? A retrolective-prolective cohort study focusing on total diagnostic yield. Eur Radiol. 1998;8(1):90-96.

78. van Rossum AB, Treurniet FE, Kieft GJ, Smith SJ, Schepers-Bok R. Role of spiral volumetric computed tomographic scanning in the assessment of patients with clinical suspicion of pulmonary embolism and an abnormal ventilation/perfusion lung scan. Thorax. 1996;51(1):23-28.

79. Wells PS, Anderson DR, Rodger M, et al. Derivation of a simple clinical model to categorize patients probability of pulmonary embolism: increasing the models utility with the SimpliRED D-dimer. Thromb Haemost. 2000;83(3):416-420.

80. Spies WG, Burstein SP, Dillehay GL, Vogelzang RL, Spies SM. Ventilation-perfusion scintigraphy in suspected pulmonary embolism: correlation with pulmonary angiography and refinement of criteria for interpretation. Radiology. 1986;159(2):383-390.

81. Mos IC, Douma RA, Erkens PM, et al; Prometheus Study Group. Diagnostic outcome management study in patients with clinically suspected recurrent acute pulmonary embolism with a structured algorithm. Thromb Res. 2014;133(6):1039-1044.

82. Sohne M, Kruip MJ, Nijkeuter M, et al; Christoper Study Group. Accuracy of clinical decision rule, D-dimer and spiral computed tomography in patients with malignancy, previous venous thromboembolism, COPD or heart failure and in older patients with suspected pulmonary embolism. $J$ Thromb Haemost. 2006;4(5):1042-1046.

83. Le Gal G, Righini M, Roy PM, et al. Value of D-dimer testing for the exclusion of pulmonary embolism in patients with previous venous thromboembolism. Arch Intern Med. 2006;166(2):176-180.

84. Anderson DR, Wells PS, Stiell I, et al. Thrombosis in the emergency department: use of a clinical diagnosis model to safely avoid the need for urgent radiological investigation. Arch Intern Med. 1999;159(5):477-482.

85. Aronen HJ, Svedström E, Yrjänä J, Bondestam S. Compression sonography in the diagnosis of deep venous thrombosis of the leg. Ann Med. 1994;26(5): 377-380.

86. Birdwell BG, Raskob GE, Whitsett TL, et al. Predictive value of compression ultrasonography for deep vein thrombosis in symptomatic outpatients: clinical implications of the site of vein noncompressibility. Arch Intern Med. 2000;160(3):309-313.

87. Cogo A, Lensing AW, Prandoni P, Büller HR, Girolami A, ten Cate JW. Comparison of real-time B-mode ultrasonography and Doppler ultrasound with contrast venography in the diagnosis of venous thrombosis in symptomatic outpatients. Thromb Haemost. 1993;70(3):404-407.

88. Friera A, Giménez NR, Caballero P, Moliní PS, Suárez C. Deep vein thrombosis: can a second sonographic examination be avoided? AJR Am J Roentgenol. 2002;178(4):1001-1005.

89. Gudmundsen TE, Vinje B, Pedersen T. Deep vein thrombosis of lower extremities. Diagnosis by real time ultrasonography. Acta Radiol. 1990;31(5): 473-475. 
90. Lensing AW, Prandoni P, Brandjes D, et al. Detection of deep-vein thrombosis by real-time B-mode ultrasonography. N Engl J Med. 1989;320(6):342-345.

91. Pedersen OM, Aslaksen A, Vik-Mo H, Bassoe AM. Compression ultrasonography in hospitalized patients with suspected deep venous thrombosis. Arch Intern Med. 1991;151(11):2217-2220.

92. Quintavalla R, Larini P, Miselli A, et al. Duplex ultrasound diagnosis of symptomatic proximal deep vein thrombosis of lower limbs. Eur J Radiol. 1992; 15(1):32-36.

93. van Ramshorst B, Legemate DA, Verzijlbergen JF, et al. Duplex scanning in the diagnosis of acute deep vein thrombosis of the lower extremity. Eur J Vasc Surg. 1991;5(3):255-260.

94. Wells PS, Anderson DR, Rodger M, et al. Evaluation of D-dimer in the diagnosis of suspected deep-vein thrombosis. N Engl J Med. 2003;349(13): 1227-1235.

95. Wells PS, Hirsh J, Anderson DR, et al. Comparison of the accuracy of impedance plethysmography and compression ultrasonography in outpatients with clinically suspected deep vein thrombosis. A two centre paired-design prospective trial. Thromb Haemost. 1995;74(6):1423-1427.

96. Wells PS, Hirsh J, Anderson DR, et al. Accuracy of clinical assessment of deep-vein thrombosis. Lancet. 1995;345(8961):1326-1330.

97. Bradley MJ, Spencer PA, Alexander L, Milner GR. Colour flow mapping in the diagnosis of the calf deep vein thrombosis. Clin Radiol. 1993;47(6): 399-402.

98. de Valois JC, van Schaik CC, Verzijlbergen F, van Ramshorst B, Eikelboom BC, Meuwissen OJ. Contrast venography: from gold standard to 'golden backup' in clinically suspected deep vein thrombosis. Eur J Radiol. 1990;11(2):131-137.

99. Habscheid W, Höhmann M, Wilhelm T, Epping J. Real-time ultrasound in the diagnosis of acute deep venous thrombosis of the lower extremity. Angiology. 1990;41(8):599-608.

100. Kalodiki E, Calahoras L, Nicolaides AN. Make it easy: duplex examination of the venous system. Phlebology. 1993;8(1):17-21.

101. Miller N, Satin R, Tousignant L, Sheiner NM. A prospective study comparing duplex scan and venography for diagnosis of lower-extremity deep vein thrombosis. Cardiovasc Surg. 1996;4(4):505-508.

102. Montefusco-von Kleist CM, Bakal C, Sprayregen S, Rhodes BA, Veith FJ. Comparison of duplex ultrasonography and ascending contrast venography in the diagnosis of venous thrombosis. Angiology. 1993;44(3):169-175.

103. Theodorou SJ, Theodorou DJ, Kakitsubata Y. Sonography and venography of the lower extremities for diagnosing deep vein thrombosis in symptomatic patients. Clin Imaging. 2003;27(3):180-183.

104. Bernardi E, Prandoni P, Lensing AW, et al; The Multicentre Italian D-dimer Ultrasound Study Investigators Group. D-dimer testing as an adjunct to ultrasonography in patients with clinically suspected deep vein thrombosis: prospective cohort study. BMJ. 1998;317(7165):1037-1040.

105. Birdwell BG, Raskob GE, Whitsett TL, et al. The clinical validity of normal compression ultrasonography in outpatients suspected of having deep venous thrombosis. Ann Intern Med. 1998;128(1):1-7.

106. Schutgens RE, Haas FJ, Biesma DH. Reduced efficacy of clinical probability score and D-dimer assay in elderly subjects suspected of having deep vein thrombosis. Br J Haematol. 2005;129(5):653-657.

107. Williams D, Lee AJ, Clark H, Webster J, Watson HG. A comparison of computerised strain gauge plethysmography with D-dimer testing in screening for deep-vein thrombosis. $\mathrm{Br} J$ Haematol. 2005;131(2):253-257.

108. Canan A, Halıcioğlu SS, Gürel S. Mean platelet volume and D-dimer in patients with suspected deep venous thrombosis. J Thromb Thrombolysis. 2012; 34(2):283-287.

109. Cornuz J, Ghali WA, Hayoz D, Stoianov R, Depairon M, Yersin B. Clinical prediction of deep venous thrombosis using two risk assessment methods in combination with rapid quantitative D-dimer testing. Am J Med. 2002;112(3):198-203.

110. D'Angelo A, D'Alessandro G, Tomassini L, Pittet JL, Dupuy G, Crippa L. Evaluation of a new rapid quantitative D-dimer assay in patients with clinically suspected deep vein thrombosis. Thromb Haemost. 1996;75(3):412-416.

111. Diamond S, Goldbweber R, Katz S. Use of D-dimer to aid in excluding deep venous thrombosis in ambulatory patients. Am J Surg. 2005;189(1):23-26.

112. Haenssle HA, Ayad N, Buhl T, et al. Limited diagnostic value of Wells-score and D-dimer testing in hospitalized dermatologic patients with symptoms of deep vein thrombosis. Eur J Dermatol. 2013;23(6):830-836.

113. Hansson PO, Eriksson H, Eriksson E, Jagenburg R, Lukes P, Risberg B. Can laboratory testing improve screening strategies for deep vein thrombosis at an emergency unit? J Intern Med. 1994;235(2):143-151.

114. Jennersjö CM, Fagerberg IH, Karlander SG, Lindahl TL. Normal D-dimer concentration is a common finding in symptomatic outpatients with distal deep vein thrombosis. Blood Coagul Fibrinolysis. 2005;16(7):517-523.

115. Knecht MF, Heinrich F. Clinical evaluation of an immunoturbidimetric D-dimer assay in the diagnostic procedure of deep vein thrombosis and pulmonary embolism. Thromb Res. 1997;88(5):413-417.

116. Le Blanche AF, Siguret V, Settegrana C, et al. Ruling out acute deep vein thrombosis by ELISA plasma D-dimer assay versus ultrasound in inpatients more than 70 years old. Angiology. 1999;50(11):873-882.

117. Luxembourg B, Schwonberg J, Hecking C, et al. Performance of five D-dimer assays for the exclusion of symptomatic distal leg vein thrombosis. Thromb Haemost. 2012;107(2):369-378.

118. Nata S, Hiromatsu S, Shintani Y, Ohno T, Akashi H, Tanaka H. D-dimer value more than $3.6 \mu \mathrm{g} / \mathrm{ml}$ is highly possible existence deep vein thrombosis. Kurume Med J. 2013;60(2):47-51. 
119. Oudega R, Moons KG, Hoes AW. Ruling out deep venous thrombosis in primary care. A simple diagnostic algorithm including D-dimer testing. Thromb Haemost. 2005;94(1):200-205.

120. Klok FA, Huisman MV, Kroft LM. Advanced Diagnostic Management of Suspected Recurrent Ipsilateral DVT With MRDTI (Theia). NCT02262052. ClinicalTrials.gov. Accessed 15 June 2018.

121. Baarslag HJ, van Beek EJ, Koopman MM, Reekers JA. Prospective study of color duplex ultrasonography compared with contrast venography in patients suspected of having deep venous thrombosis of the upper extremities [published correction appears in Ann Intern Med. 2003;138(5):438]. Ann Intern Med. 2002;136(12):865-872.

122. Baxter GM, Kincaid W, Jeffrey RF, Millar GM, Porteous C, Morley P. Comparison of colour Doppler ultrasound with venography in the diagnosis of axillary and subclavian vein thrombosis. Br J Radiol. 1991;64(765):777-781.

123. Haire WD, Lynch TG, Lund GB, Lieberman RP, Edney JA. Limitations of magnetic resonance imaging and ultrasound-directed (duplex) scanning in the diagnosis of subclavian vein thrombosis. J Vasc Surg. 1991;13(3):391-397.

124. Kleinjan A, Di Nisio M, Beyer-Westendorf J, et al. Safety and feasibility of a diagnostic algorithm combining clinical probability, D-dimer testing, and ultrasonography for suspected upper extremity deep venous thrombosis: a prospective management study. Ann Intern Med. 2014;160(7):451-457.

125. Köksoy C, Kuzu A, Kutlay J, Erden I, Ozcan H, Ergîn K. The diagnostic value of colour Doppler ultrasound in central venous catheter related thrombosis. Clin Radiol. 1995;50(10):687-689.

126. Prandoni P, Polistena P, Bernardi E, et al. Upper-extremity deep vein thrombosis. Risk factors, diagnosis, and complications. Arch Intern Med. 1997; 157(1):57-62.

127. Sottiurai VS, Towner K, McDonnell AE, Zarins CK. Diagnosis of upper extremity deep venous thrombosis using noninvasive technique. Surgery. 1982; 91(5):582-585.

128. Merminod T, Pellicciotta S, Bounameaux H. Limited usefulness of D-dimer in suspected deep vein thrombosis of the upper extremities. Blood Coagul Fibrinolysis. 2006;17(3):225-226.

129. Sartori M, Migliaccio L, Favaretto E, et al. D-dimer for the diagnosis of upper extremity deep and superficial venous thrombosis. Thromb Res. 2015; 135(4):673-678.

130. Bates SM, Jaeschke R, Stevens SM, et al. Diagnosis of DVT: Antithrombotic Therapy and Prevention of Thrombosis, 9th ed: American College of Chest Physicians Evidence-Based Clinical Practice Guidelines. Chest. 2012;141(suppl 2):e351S-e418S.

131. Schünemann HJ, Wiercioch W, Brozek J, et al. GRADE Evidence to Decision (EtD) frameworks for adoption, adaptation, and de novo development of trustworthy recommendations: GRADE-ADOLOPMENT. J Clin Epidemiol. 2017;81:101-110. 